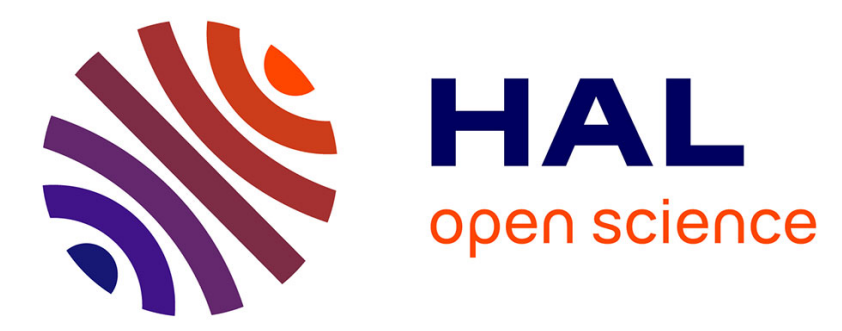

\title{
Spatially dissociated intracerebral maps for face- and house-selective activity in the human ventral occipito-temporal cortex
}

Simen Hagen, Corentin Jacques, Louis Maillard, Sophie Colnat-Coulbois, Bruno Rossion, Jacques Jonas

\section{To cite this version:}

Simen Hagen, Corentin Jacques, Louis Maillard, Sophie Colnat-Coulbois, Bruno Rossion, et al.. Spatially dissociated intracerebral maps for face- and house-selective activity in the human ventral occipito-temporal cortex. Cerebral Cortex, 2020, 30 (7), pp.4026-4043. 10.1093/cercor/bhaa022 . hal-02931203

\section{HAL Id: hal-02931203 \\ https://hal.science/hal-02931203}

Submitted on 2 Dec 2020

HAL is a multi-disciplinary open access archive for the deposit and dissemination of scientific research documents, whether they are published or not. The documents may come from teaching and research institutions in France or abroad, or from public or private research centers.
L'archive ouverte pluridisciplinaire HAL, est destinée au dépôt et à la diffusion de documents scientifiques de niveau recherche, publiés ou non, émanant des établissements d'enseignement et de recherche français ou étrangers, des laboratoires publics ou privés. 


\section{Spatially dissociated intracerebral maps for face- and house-selective activity in the human ventral occipito-temporal cortex}

Simen Hagen ${ }^{1}$, Corentin Jacques ${ }^{2}$, Louis Maillard ${ }^{1,3}$, Sophie Colnat-Coulbois ${ }^{1,4}$, Bruno

$$
\text { Rossion }^{1,2,3} \text {, Jacques Jonas }{ }^{1,3}
$$

${ }^{1}$ Université de Lorraine, CNRS, CRAN, F-54000 Nancy, France

${ }^{2}$ Psychological Sciences Research Institute and Institute of Neuroscience, University of Louvain, B1348 Louvain-La-Neuve, Belgium

${ }^{3}$ Université de Lorraine, CHRU-Nancy, Service de Neurologie, F-54000 Nancy, France

${ }^{4}$ Université de Lorraine, CHRU-Nancy, Service de Neurochirurgie, F-54000 Nancy, France

\section{Corresponding Author:}

Bruno Rossion

CRAN UMR 7039, CNRS - Universite de Lorraine,

2 Avenue de la forêt de Haye

54516 Vandoeuvre-lès-Nancy, France.

Tel: +33 (0) 383858053

E-mail: bruno.rossion@univ-lorraine.fr 


\section{Abstract}

We report a comprehensive mapping of the human ventral occipito-temporal cortex (VOTC) for selective responses to frequency-tagged faces or landmarks (houses) presented in rapid periodic trains of objects, with intracerebral recordings in a large sample $(\mathrm{N}=75)$. Faceselective contacts are three times more numerous than house-selective contacts and show a larger amplitude, with a right hemisphere advantage for faces. Most importantly, these category-selective contacts are spatially dissociated along the lateral-to-medial VOTC axis, respectively, consistent with neuroimaging evidence. At the minority of "overlap" contacts responding selectively to both faces and houses, response amplitude to the two categories is not correlated, suggesting a contribution of distinct populations of neurons responding selectively to each category. The medio-lateral dissociation also extends into the underexplored anterior temporal lobe (ATL). In this region, a relatively high number of intracerebral recording contacts show category-exclusive responses (i.e., without any response to baseline visual objects) to faces but rarely to houses, in line with the proposed role of this region in processing people-related semantic information. Altogether, these observations shed novel insight on the neural basis of human visual recognition and strengthen the validity of the frequency-tagging approach coupled with intracerebral recordings in temporal epileptic patients to understand human brain function.

Key Words: Face categorization, frequency-tagging, SEEG, fusiform gyrus, anterior temporal lobe 


\section{Introduction}

The human visual system is highly efficient at transforming retinal input into meaningful categories, as exemplified by the ease of detecting highly variable human faces among other variable nonface objects (i.e., generic face categorization). Categorizing visual stimuli as faces is a challenging brain function since, besides the inherent variability in face and nonface object identity, each instance of a face or an object can produce an infinite number of retinal inputs, resulting from changes in e.g., size (distance), position, lighting, or orientation. The neural basis of human face categorization has been studied extensively in functional magnetic resonance imaging (fMRI) studies, which consistently report larger responses to faces in the lateral parts of the middle fusiform gyrus (latFG) and in the inferior occipital gyrus (IOG) of the ventral occipito-temporal cortex (VOTC) (Puce et al. 1995; Kanwisher et al. 1997; Haxby et al. 2000; see Grill-Spector et al. 2017 for recent review).

These indirect measures of face-selective neural activity can be complemented by direct intracranial recordings of brain activity in human epileptic patients (Allison et al. 1994; Allison et al. 1999; Halgren et al. 1994; Vidal et al. 2010; Ghuman et al. 2014; Engell and McCarthy 2014; Jonas et al. 2016; Kadipasaoglu et al. 2016; see Rossion et al. 2018 for review). These investigations are valuable by the high temporal resolution that they afford but also because they provide direct neural measures without substantial regional variations in signal-to-noise ratio. Moreover, they provide direct links with the causal effects of electrical stimulation (e.g., Jonas et al. 2012; Parvizi et al. 2012). However, these investigations remain rare and are often limited in terms of the number of individual brains sampled as well as their spatial range of investigation, which is determined by strict clinical criteria. Moreover, the exact location/source of the neurophysiological recordings remain uncertain (Katzner et al. 2009; Herreras 2016). This important approach for human neuroscience research therefore 
requires further validation by testing large cohort of patients with objective quantification of responses of interest across wide cortical regions, documenting similarities and differences with other measures (Rossion et al. 2018).

In this context, a recent study isolated face categorization responses with a frequencytagging approach (Fast Period Visual Stimulation [FPVS]) applied to 28 patients implanted with intracerebral (i.e., depth) electrodes across the VOTC (Jonas, Jacques, et al., 2016). Although face-selective responses were found across the whole VOTC, replicating previous intracranial observations with more traditional stimulation approaches (e.g., Allison et al., 1999), clusters of high-amplitude face-selective contacts with a peak in the right lateral section of the middle fusiform gyrus were found, supporting fMRI observations (i.e., evidence for the "Fusiform Face Area", "FFA" with a direct neural measure). Moreover, face-selective responses were found in several regions of the ventral anterior temporal lobe (ATL), an underexplored region in fMRI due to severe magnetic susceptibility artefacts (Wandell, 2011; Axelrod \& Yovel, 2013; Rossion et al., 2018). Notably, up to $40 \%$ of population-level neural responses in the ATL showed responses to faces without any significant response to nonface objects (i.e., 'face-exclusive' contacts; Jonas et al., 2016).

Although this frequency-tagging approach has substantial advantages in terms of sensitivity, objectivity, and quantification, these observations raise at least two important unresolved issues. First, to what degree does the wide distribution of face-selective responses in the human VOTC reflect a lack of specificity of this approach? That is, could this wide distribution be due to the recording of neural activity from distant sources in intracerebral recordings (Herreras 2016; Dubey \& Ray, 2019), or to the additional contribution of non category-selective responses triggered by the mere periodic repetition of the same kinds of items in a continuous stream of variable nonface objects, or else to a combination of these 
two factors? Second, are face-exclusive contacts found predominantly in the ATL truly category-selective - in particular limited to faces due to their rich network of semantic associations (Collins et al., 2016; Rice et al., 2018) - or do they rather reflect some kind of generic "oddball" responses to rare periodic events in such stimulation sequences? These issues are important to resolve not only to inform about the neural basis of face categorization and visual categorization in general, but also to better understand the validity and limitations of the frequency-tagging approach in the context of human intracerebral recordings.

The current study directly addresses these issues in a very large group of individual human brains $(N=75)$ implanted with intracerebral electrodes. Subjects viewed variable objects images presented periodically at $6 \mathrm{~Hz}$ with either variable face- or house-images interleaved as every 5th image in separate sequences (Figure 1). Hence, both face-selective and house-selective neural responses were objectively identified and quantified at the facestimulation frequency $(6 \mathrm{~Hz} / 5=1.2 \mathrm{~Hz})$ and harmonics in different stimulation sequences. Pictures of houses provide a robust test to the issues at stake because it is known that houses, as spatial landmarks or places, are associated with responses in the medial regions of the VOTC such as the collateral sulcus, the lingual sulcus and the parahippocampal gyrus (Epstein \& Kanwisher, 1998; Weiner \& Grill-Spector, 2010; Epstein, 2014; Kadipasoglou et al., 2016). Moreover, faces and houses evoke quantitatively and qualitatively different category-selective responses in scalp EEG (Jacques et al., 2016). Hence, we hypothesized that face- and house-selective responses (i.e., discriminating faces or houses from a variety of other visual objects) would elicit largely spatially distinct maps in the VOTC, with a predominance in lateral and medial temporal regions respectively. Moreover, we took advantage of an extensive sampling of the ATL to test whether this spatial dissociation extends to these anterior regions. Finally, based on growing evidence for the role of the ATL 
in person recognition and semantics (e.g., Gainotti, 2013; Rice et al., 2018), we hypothesized exclusive responses in the ATL to be truly category-specific, and with no or little response to houses on these contacts. Such findings would not only shed important insight on the neural basis of human face categorization but would also strengthen the validity and the frequencytagging approach combined with human intracerebral recordings in temporal epileptic patients to understand brain function.

\section{Materials and Methods}

\section{Participants}

The study included 75 patients ( 35 females, mean age: $32.3 \pm 8.4$ years, 68 righthanded) undergoing clinical intracerebral evaluation with depth electrodes (StereoElectroEncephaloGraphy, SEEG) for refractory partial epilepsy, studied in the Epilepsy Unit of the University Hospital of Nancy between 2013 and 2017. Patients were included in the study if they had at least one intracerebral electrode implanted in the VOTC (Figure 1C). The data on periodic faces from twenty-eight of the 75 patients were included in the study of Jonas et al. (2016). They all gave written consent to participate to the study, which was part of a protocol approved by the Ethics committee of the University Hospital of Nancy.

\section{Intracerebral electrode implantation and recording}

Intracerebral electrodes were stereotactically implanted within the participants' brains for clinical purposes, i.e., to delineate their seizure onset zones and to functionally map the surrounding cortex in the perspective of an eventual epilepsy surgery (Bédos Ulvin et al. 2017). Each $0.8 \mathrm{~mm}$ diameter intracerebral electrode contains 8-15 independent recording contacts of $2 \mathrm{~mm}$ in length separated by $1.5 \mathrm{~mm}$ from edge to edge (for details about the electrode implantation procedure, see Salado et al. 2017). Intracerebral EEG was sampled at 
a $512 \mathrm{~Hz}$ with a 256-channel amplifier and referenced to either a midline prefrontal scalp electrode (FPz, available in 66 participants) or an intracerebral contact in the white matter (in 9 participants). The data were not re-referenced. Contacts located in brain lesions visible on structural MRI were excluded from any analysis ( $n=67 ; 1.83 \%$ of all contacts).

\section{Fast periodic visual stimulation paradigm}

Stimuli. We used 200 grayscale natural images of various non-face objects (from 14 non-face categories: cats [n=9], dogs [n=5], horses [n=5], birds [n=24], flowers [n=15], fruits [n=28], vegetables [n=21], houseplants [n=15], phones [n=13], chairs [n=15], cameras $[n=6]$,

dishes [n=15], guitars [n=15], lamps [n=14]), 50 grayscale natural images of faces and 50 grayscale natural images of houses (see Figure 1 for examples of stimuli). Each image contained an unsegmented object, face or house near the center, which differed in terms of size, viewpoint, lighting conditions and background. Images were equalized for mean pixel luminance and contrast (i.e. standard deviation across pixels). Faces (or houses) represented $20 \%$ of the stimuli. Since, they appeared 1 out of five stimuli $(20 \%)$, images of faces (or houses) were repeated the same amount of time than images of nonface objects. In a recording sequence, there were 227 cycles displaying objects, and at each cycle an image was sampled randomly from the 200 object stimuli. In contrast, there were 75 cycles displaying faces or houses, and at each cycle an image was sampled randomly from the 50 face or 50 house stimuli, respectively.

Experimental procedure. Participants viewed continuous sequences of natural images of objects presented at a fast rate of $6 \mathrm{~Hz}$ through sinusoidal contrast modulation. In separate sequences, images of either faces or houses were presented periodically as every 5th stimulus so that the frequency of face/house presentation was $1.2 \mathrm{~Hz}$ (i.e. $6 \mathrm{~Hz} / 5)$ (see Figure 1, see also Videos 1 and 2 for an example of visual stimulation). All images were 
randomly selected from their respective categories. A sequence lasted $70 \mathrm{~s}: 66 \mathrm{~s}$ of stimulation at full-contrast flanked by $2 \mathrm{~s}$ of fade-in and fade-out, where contrast gradually increased or decreased, respectively. During one sequence, there were 75 presentations of faces or houses and 227 presentations of objects. During the sequences, participants were instructed to fixate a small black cross which was presented continuously at the center of the stimuli and to detect brief (500 ms) color-changes (black to red) of this fixation-cross. All but 19 participants saw the same number of sequences of face and houses (minimum 1 sequence per condition). The average number of sequences across all patients was 2.67 and 2.37 of face- and house-sequences, respectively. No participant had seizures in the 2 hours preceding FPVS recordings. 
A
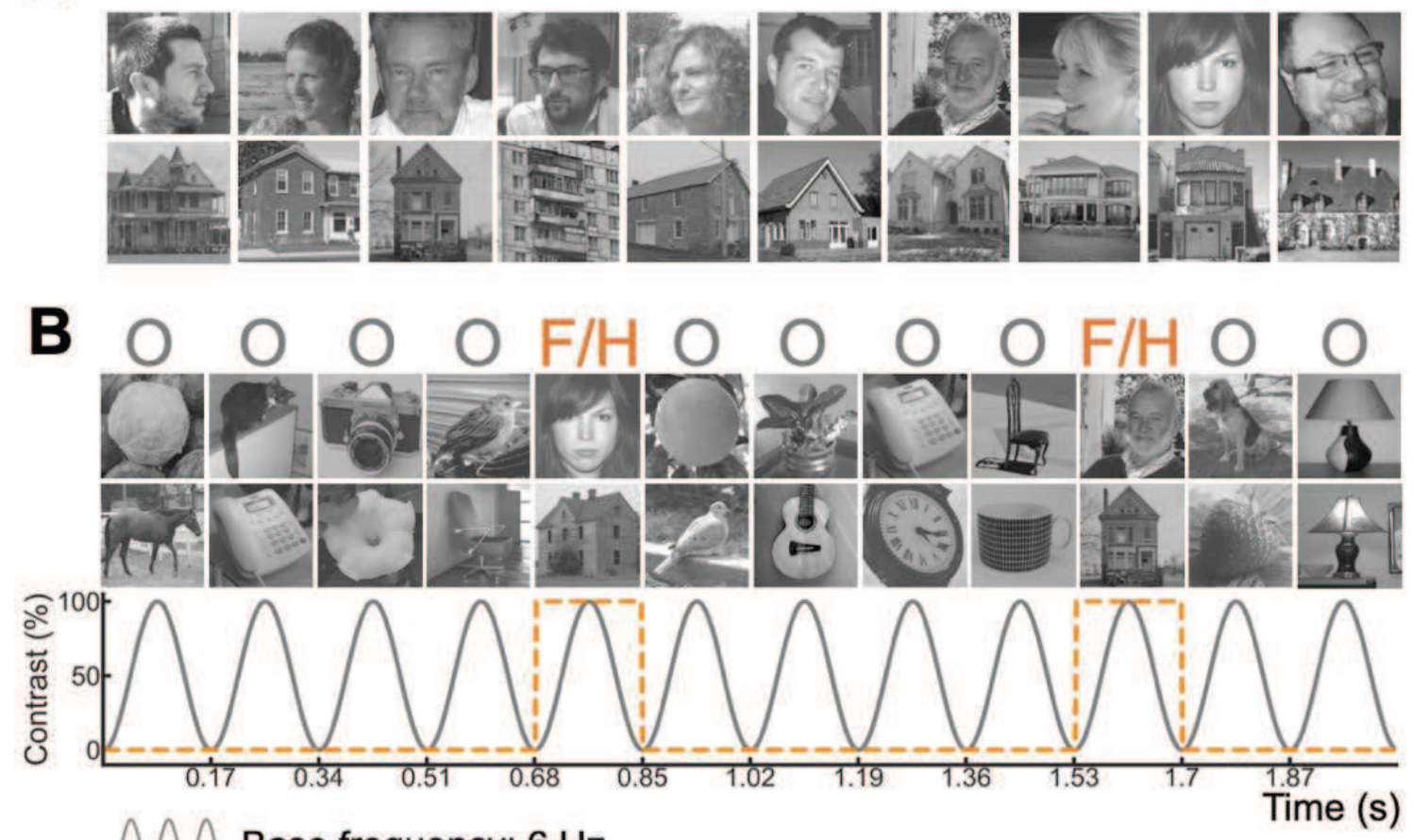

М Base frequency: $6 \mathrm{~Hz}$

Face- and house-selective frequency: $6 \mathrm{~Hz} / 5=1.2 \mathrm{~Hz}$

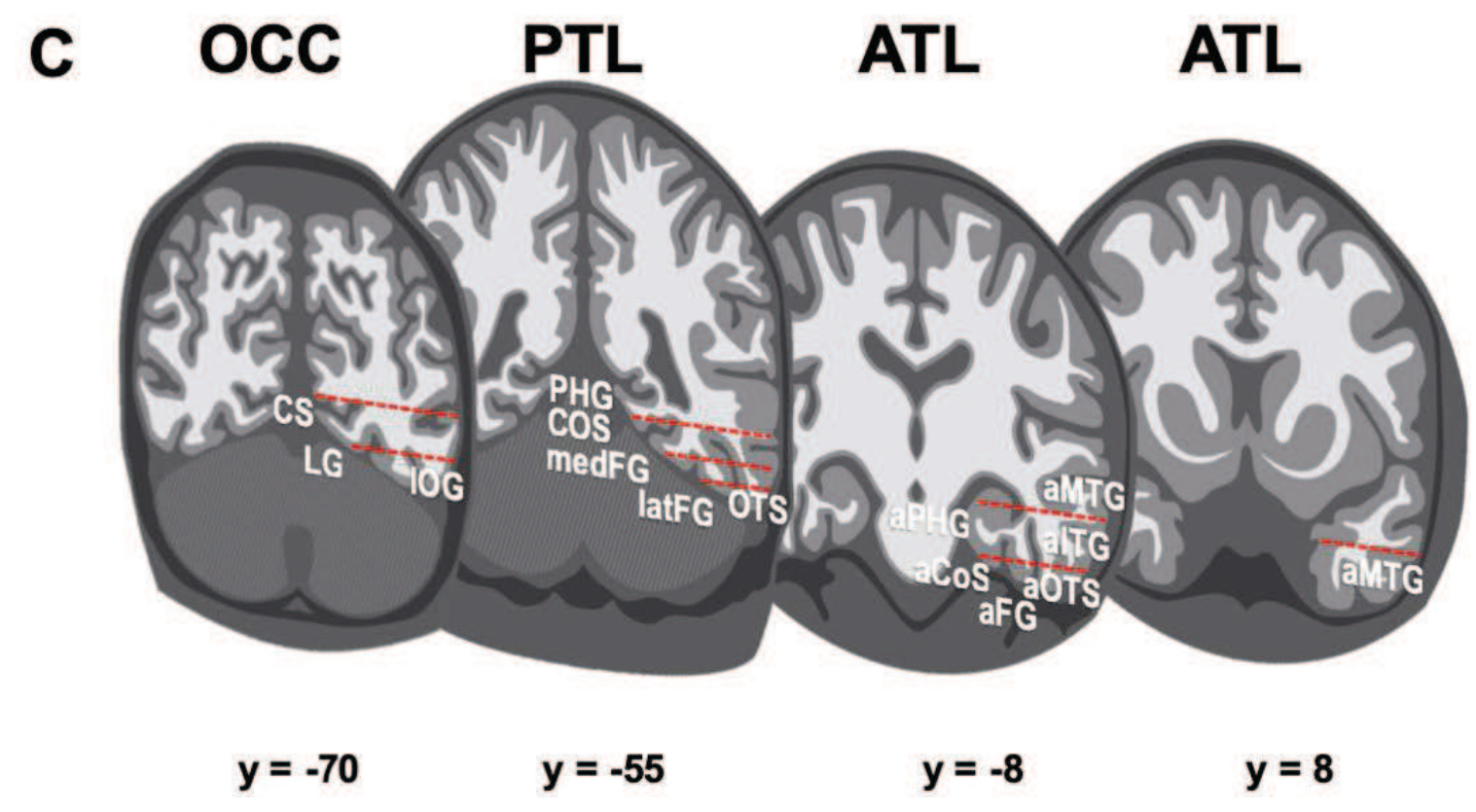

Figure 1. FPVS and SEEG methods. A. Example of natural images of faces and houses used in the experiment (actual face images not shown for copyright reasons). B. Images of living or non-living objects were presented by sinusoidal contrast modulation at a rate of 6 
stimuli per second (6 Hz) with different images of either faces or houses presented in separate sequences every 5 stimuli (i.e., appearing at the frequency of $6 \mathrm{~Hz} / 5=1.2 \mathrm{~Hz}$ ). $\boldsymbol{C}$. Schematic coronal representation of the typical trajectories of depth electrodes implanted in the VOTC (adapted from Jonas et al., 2016). Electrodes consist of 8-15 contiguous recording contacts (red rectangles) spread along the electrode length, along the medio-lateral axis. Acronyms: ATL: anterior temporal lobe; PTL: posterior temporal lobe; OCC: occipital lobe; PHG: parahippocampal gyrus; CoS: collateral sulcus; FG: fusiform gyrus; ITG: inferior temporal gyrus; MTG: middle temporal gyrus; OTS: occipito-temporal sulcus; CS: calcarine sulcus; IOG: inferior occipital gyrus; LG: lingual gyrus; a: anterior; lat: lateral; med: medial.

Frequency domain processing. Signals corresponding to the face and house conditions were processed the same way. Segments of SEEG corresponding to stimulation sequences were extracted (74-second segments, $-2 \mathrm{~s}$ to $+72 \mathrm{~s}$ ). The $74 \mathrm{~s}$ data segments were cropped to contain an integer number of $1.2 \mathrm{~Hz}$ cycles beginning $2 \mathrm{~s}$ after the onset of the sequence (right at the end of the fade-in period) until approximately $65 \mathrm{~s}$, i.e. before stimulus fade-out ( 75 face cycles $\approx 63 \mathrm{~s}$ ). No artifact rejection was performed since EEG artifacts generate broadband noise in the frequency domain that locate mostly outside the narrow frequency bins containing the signal measurements $(1.2 \mathrm{~Hz}$ and associated harmonics; Regan, 1989; Jonas et al., 2016). Sequences of recorded voltage (i.e., timedomain) were averaged separately for each participant and condition. Averaging sequences in the time-domain before the Fast Fourier Transform (FFT) increases signal-to-noise ratio by cancelling out non-phase locked noise. Subsequently, FFT was applied to the full length of the cropped averaged time sequences. The amplitude spectra were extracted for all contacts by taking the modulus of the Fourier coefficients at each frequency bin normalized (by 
dividing) by half of the number of time samples in the time series. No normalization was performed on the time series prior to running the FFT. The long recording sequence resulted in a spectrum with a high frequency resolution of 0.0159 (1/63sec). No data segments were excluded from the analysis. No other processing was performed to the data.

Selective responses. The FPVS approach used here allows identifying and separating two distinct types of responses in both conditions: (1) a base response occurring at the base stimulation frequency $(6 \mathrm{~Hz})$ and its harmonics, as well as (2) a selective response at $1.2 \mathrm{~Hz}$ and its harmonics (face-selective or house-selective response). In both face and house conditions, selective responses significantly above noise level at the face/house frequency $(1.2 \mathrm{~Hz})$ and its harmonics were determined as follows: (1) the FFT spectrum was cut into 4 segments centered at the face/house frequency and harmonics, from the 1st until the 4th $(1.2 \mathrm{~Hz}$ until $4.8 \mathrm{~Hz})$, and surrounded by 25 neighboring bins on each side (Figure 2A); (2) the amplitude values in these 4 segments of FFT spectra were summed (Figure 2B); (3) the summed FFT spectrum was transformed into a Z-score (Figure 2C). Zscores were computed as the difference between the amplitude at the face/house frequency bin and the mean amplitude of 48 surrounding bins ( 25 bins on each side, excluding the 2 bins directly adjacent to the bin of interest, i.e., 48 bins) divided by the standard deviation of amplitudes in the corresponding 48 surrounding bins. A contact was considered as showing a selective response in a given condition if the Z-score at the frequency bin of face or house stimulation exceeded 3.1 (i.e., $p<0.001$ one-tailed: signal $>$ noise). 

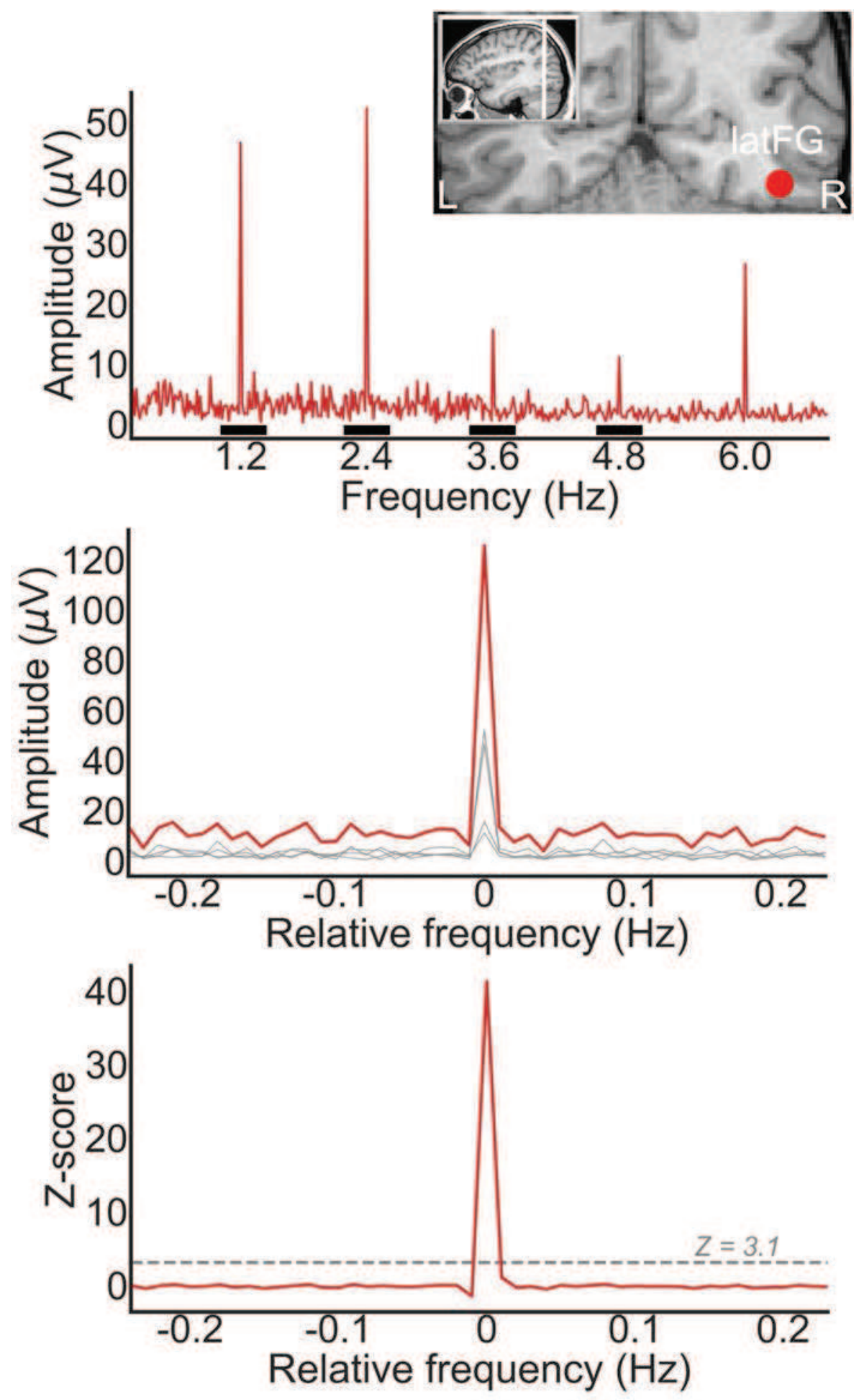

Figure 2. Intracerebral selective responses recorded in the VOTC. A. Intracerebral EEG frequency-domain responses recorded at an individual recording contact (raw FFT amplitude) located in the right latFG in a single participant during a face sequence. The anatomical location of the contact is shown in a coronal MRI slice. Face-selective responses are 
observed at $1.2 \mathrm{~Hz}$ and harmonics. B. Significant face-selective responses were determined by first segmenting the FFT spectrum into four segments centered at the frequency of face stimulation and its harmonics up to $4.8 \mathrm{~Hz}$ (i.e., 1.2, 2.4, 3.6, and 4.8 Hz). Individual FFT segments are shown in gray (see horizontal gray bars on the $X$ axis in $A$, representing the length of each FFT segment). The four segments, containing both the signal and the surrounding noise, were then summed (dark gray line). The 0 mark corresponds to the face stimulation frequency. C. Z-score transformation of the summed FFT spectrum for statistical purpose. The $Z$ score at the face frequency exceeds $3.1(p<0.001)$, indicating that this contact shows a significant face-selective response. The same procedure was used to identify house-selective responses.

Classification of significant contacts. Based on the pattern of discrimination responses across the 2 conditions (i.e., significant or not), we labeled each significant contact as follows: (i) contacts showing a significant face discrimination response, but not a significant house discrimination response, were defined as "face" (+face, -house); (ii) contacts showing a significant house response, but not a significant face discrimination response, were defined as "house" (-face, +house); and (iii) contacts showing significant discrimination responses to both faces and houses, were defined as "overlap" (+face, +house).

Quantification of response amplitude. Baseline-corrected amplitudes were computed as the difference between the amplitude at each frequency bin and the average of 48 corresponding surrounding bins (up to 25 bins on each side, i.e., 50 bins, excluding the 2 bins directly adjacent to the bin of interest, i.e., 48 bins). Face- and house selective responses were quantified separately as the sum of the baseline-subtracted amplitudes at the face/house frequency from the 1st until the 14th $(1.2 \mathrm{~Hz}$ until $16.8 \mathrm{~Hz})$, excluding the 5th and 
10th harmonics $(6 \mathrm{~Hz}$ and $12 \mathrm{~Hz})$ that coincided with the base frequency (Jonas et al. 2016). Base response amplitudes were quantified separately as the sum of the baseline-subtracted amplitudes at the base frequency from the 1st until the 4th (6 Hz until $24 \mathrm{~Hz}$ ), separately for face and house sequences.

\section{Contact Localization in the Individual Anatomy}

The exact position of each contact in the individual anatomy was determined by fusing the postoperative CT scan with a T1-weighted MRI. Contacts inside the gray matter were anatomically labeled in the individual anatomy using the same topographic VOTC parcellation as in Jonas et al. (2016) and Lochy et al. (2018) based on anatomical landmarks (Figure S1). Major VOTC sulci (collateral sulcus and occipito-temporal sulcus) served as medio-lateral divisions. Postero-anterior divisions were the anterior tip of the parieto-occipital sulcus for the border between occipital and temporal lobes, and the posterior tip of the hippocampus for the border between the posterior temporal lobe (PTL) and the anterior temporal lobe (ATL) (Kim et al., 2000). We did not include the temporal pole in our analyses (i.e., contacts anterior to the limen insulae, Ding et al. 2009). Therefore, we considered contacts in the ATL if they were located anteriorly to the posterior tip of the hippocampus and posterior to the limen insulae.

\section{Proportion and amplitude maps in MNI space}

In a separate analysis, anatomical MRIs were spatially normalized to determine Talairach coordinates of intracerebral contacts (for transformation procedure, see Koessler et al., 2009). Talairach coordinates of the intracerebral contacts were used to perform group analyses and visualization. The cortical surface used to display group maps was obtained from segmentation of the Collin27 brain from AFNI (Cox, 1996) which is aligned to the Talairach space. Using Talairach coordinates, we computed the local proportion and amplitudes of the discrimination intracerebral contacts across the VOTC. Local proportion and 
amplitudes of contacts were computed in volumes (i.e., "voxels") of size $15 \times 15 \times 200 \mathrm{~mm}$ (for the $\mathrm{X}$, left-right; $\mathrm{Y}$, posterior-anterior; and Z, inferior-superior dimensions, respectively) by steps of $3 \times 3 \times 200 \mathrm{~mm}$ over the whole VOTC. A large voxel size in the $Z$ dimension was used to collapse across contacts along the inferior-superior dimension. For each voxel, we extracted the following information across all participants in our sample: (i) the number of recorded contacts located within the voxel; (ii) the number of contacts showing a significant response for each type of discrimination; and (iii) the mean amplitudes in the significant contacts. For each voxel and each type of discrimination (i.e. face, house, overlap), we computed the proportion of significant contacts over recorded contacts/ (proportions are crucial here since sampling differs across regions), as well as the mean amplitudes over/in the significant contacts. To ensure reliability and reproducibility, we only considered voxels in which at least two participants showed significant responses. Then, for each voxel, we determined whether the proportion/amplitudes of significant contacts was significantly above zero using a bootstrap procedure in the following way: (i) sampling contacts from the voxel (the same number as the number of recorded contacts in the voxel) with replacement; (ii) determining the proportion of significant contacts for this bootstrap sample and storing this value; (iii) repeating steps $\mathrm{i}$ and ii 5,000 times to generate a distribution of bootstrap proportions and to estimate the $p$-value as the fraction of bootstrap proportions equal to zero.

\section{Bayesian estimation}

Bayesian estimation was used as a statistical tool for direct comparisons between conditions (for information on Bayesian estimation, see Krutche, 2013, 2014). This method assigns credibility to different parameter values (e.g., parameter $=$ mean difference of face and house amplitudes in latFG) according to their consistency with the data. The more consistent the data is with a parameter value, the more credible that parameter value is (and 
vice versa for parameter values not consistent with the data). The posterior probability distribution expresses the probability of parameter values given the data, from which we computed $95 \%$ high density confidence interval (95\% HDI), reflecting the 95\% most likely parameter values given the data. A separate decision criterion was applied to yield a discrete decision as to whether there was evidence for a difference larger than 0. Specifically, a 95\% HDI excluding a difference of 0 was interpreted as sufficient evidence for a difference (i.e., 0 was not among the $95 \%$ most likely values given the data), whereas a 95\% HDI including a difference of 0 was interpreted as insufficient evidence for a difference (i.e., 0 was among the $95 \%$ most likely values given the data).

Continuous data (e.g., amplitudes, Talairach coordinates) was described by a normal distribution with the parameters mean and standard deviation. A noninformative prior distribution was specified by a uniform distribution. All dichotomous data (described as proportions) was described by a binominal distribution with the parameter theta. A noninformative prior distribution was specified using a beta distribution with shape parameters $a=1$ and $b=1$. A No-U-Turn sampler (NUTS) Marcov Chain Monte Carlo (MCMC) algorithm, implemented using the Pystan package (Carpenter et al. 2017) in the Python programming language, was used to estimate the posterior probability distribution. For each estimation, four chains were initialized, and each provided 100,000 samples. The burn-in period consisted of 50,000 samples. The chains were checked for convergence by inspecting the overlap of the different chains as plotted in trace plots and density plots of the parameter values sampled in each chain.

\section{Results}

Following Fourier Transform of SEEG data, high SNR selective responses (for faces and/or houses) were identified in the VOTC exactly at the face/house presentation frequency 
(1.2 Hz) and harmonics (see Figure 2A for an example in the face condition). Significant selective responses were determined based on a combination of the first four harmonics (i.e. 1.2, 2.4, 3.6 and 4.8 Hz, Figure 2B) and a z-score transform $(z>3.1, p<0.001$, Figure $2 \mathrm{C})$.

We found 1154 contacts with category-selective responses (for faces and/or houses) in 71 participants (among 3588 contacts implanted in the VOTC of 75 participants; i.e., $32 \%$ of recorded contacts). Among these contacts, $46.6 \%$ were selective to faces only ("face" contacts, $537 / 1154$, participants $=65$ ) and $15.8 \%$ were selective to houses only ("house" contacts, $182 / 1154$, participants $=47$ ). The remaining $37.7 \%$ contacts were selective to both faces and houses ("overlap" contacts, 435/1154, participants $=54$ ). An example of the response profile of each contact type is shown in Figure 3. Each contact was localized in the individual anatomy using a topographic parcellation of the VOTC and in the MNI space to perform group analyses and visualization (Figure 3; see Lochy et al. 2018 for the parcellation; Table 1 for contact count by hemisphere and region). 
Group maps
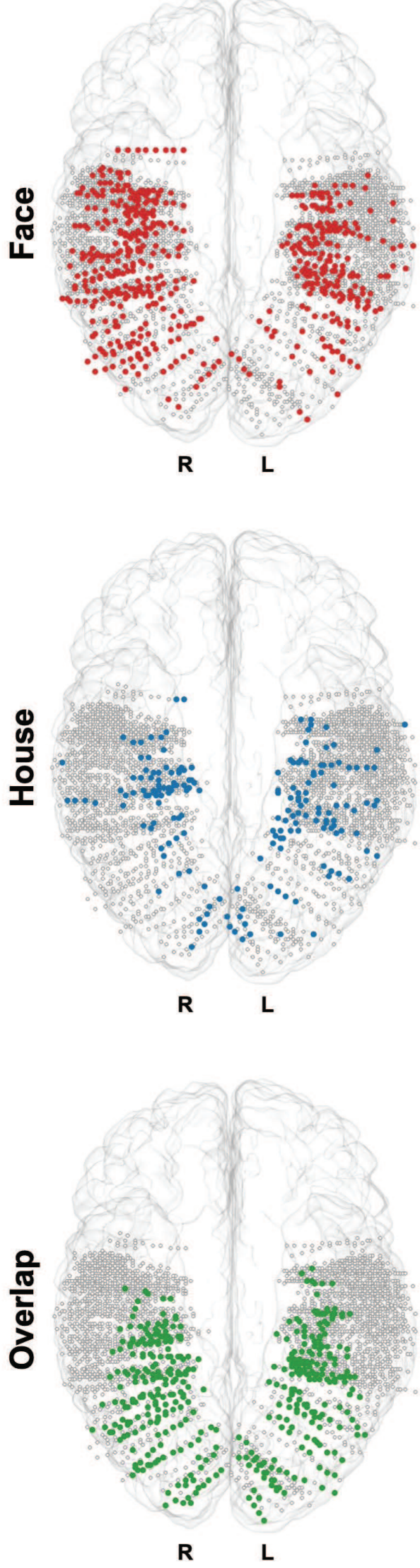

Sample contacts
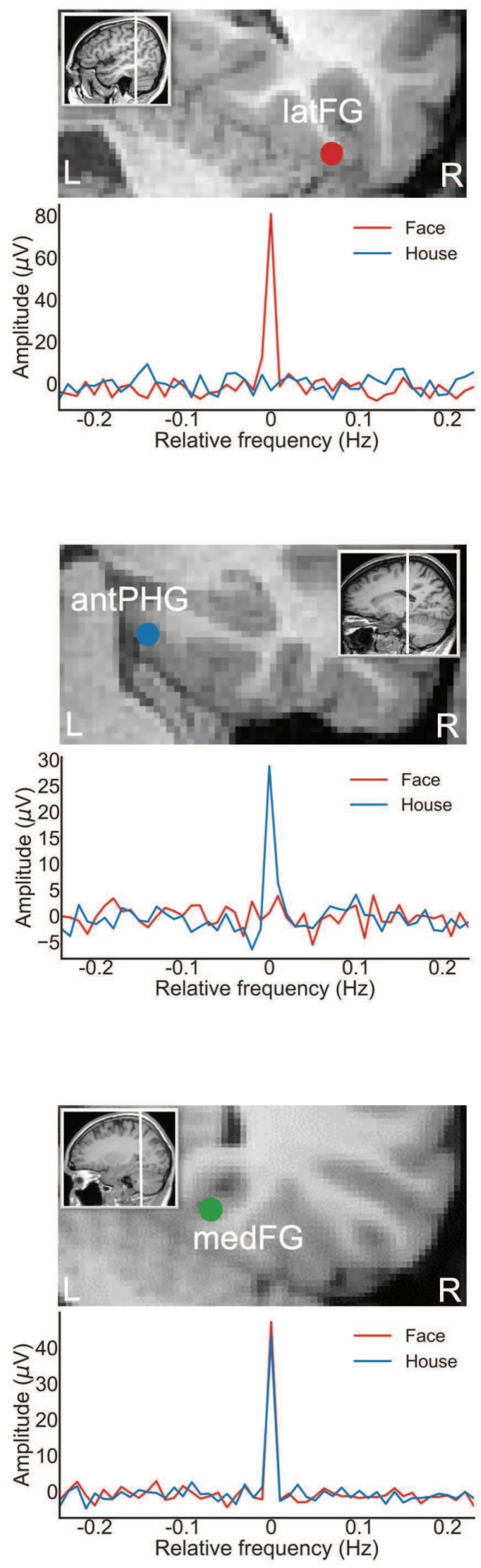
Figure 3. Classification and distribution of three types of selective contacts. Left. Maps of all 3588 VOTC recording contacts across the 75 individual brains displayed in the MNI space using a transparent reconstructed cortical surface of the Colin27 brain. Each circle represents a single contact. Color filled circles correspond to selective contacts colored according to their category (face, house, overlap). White-filled circles correspond to contacts on which no selective responses were recorded. Right. Examples of baseline corrected FFT spectra for each contact type (3 individual contacts in 3 different participants). Top: face contact, selective to faces only, middle: house contact selective to houses only; bottom: overlap contact selective to both faces and houses. Their anatomical location is illustrated in the respective coronal MRI slices. The letters below each hemisphere $(R, L)$ refers to the hemispheric side. 
Table 1. Number of selective contacts and corresponding number of participants (in parentheses) in each anatomical region. Acronyms: ATL: anterior temporal lobe; PTL: posterior temporal lobe; OCC: occipital lobe; VMO: ventromedial occipital; IOG: inferior occipital gyrus; PHG: parahippocampal gyrus; FG: fusiform gyrus; MTG: middle temporal gyrus; ITG: inferior temporal gyrus; OTS: occipito-temporal sulcus; COS: collateral sulcus; a: anterior; lat: lateral; med: medial.

\begin{tabular}{|l|l|l|l|l|l|l|}
\hline & \multicolumn{4}{|l}{ Left Hemisphere } & \multicolumn{3}{l|}{ Right Hemisphere } \\
\hline Regions & Faces & Houses & Overlap & Faces & Houses & Overlap \\
\hline VMO & $14(4)$ & $15(6)$ & $49(7)$ & $25(6)$ & $13(6)$ & $33(4)$ \\
\hline IOG & $15(8)$ & $5(2)$ & $22(8)$ & $37(11)$ & $0(0)$ & $39(11)$ \\
\hline Total OCC & $\mathbf{2 9}(8)$ & $\mathbf{2 0 ( 6 )}$ & $\mathbf{7 1 ( 9 )}$ & $\mathbf{6 2 ( 1 3 )}$ & $\mathbf{1 3 ( 6 )}$ & $\mathbf{7 2 ( 1 2 )}$ \\
\hline PHG & $0(0)$ & $1(1)$ & $2(1)$ & $0(0)$ & $5(4)$ & $1(1)$ \\
\hline medFG & $12(8)$ & $23(11)$ & $55(19)$ & $17(10)$ & $6(3)$ & $48(14)$ \\
\hline latFG & $34(16)$ & $10(8)$ & $23(11)$ & $43(15)$ & $2(2)$ & $32(13)$ \\
\hline MTG/ITG & $27(8)$ & $6(4)$ & $5(4)$ & $27(10)$ & $5(1)$ & $3(1)$ \\
\hline TOTAL PTL & $\mathbf{7 3 ( 1 9 )}$ & $\mathbf{4 0 ( 1 6 )}$ & $\mathbf{8 5 ( 2 0 )}$ & $\mathbf{8 7 ( 2 1 )}$ & $\mathbf{1 8 ( 8 )}$ & $\mathbf{8 4}(18)$ \\
\hline antPHG & $0(0)$ & $7(6)$ & $1(1)$ & $0(0)$ & $6(4)$ & $0(0)$ \\
\hline antCOS & $48(23)$ & $17(10)$ & $26(17)$ & $41(16)$ & $36(18)$ & $31(14)$ \\
\hline antFG & $8(5)$ & $2(1)$ & $7(5)$ & $7(3)$ & $2(1)$ & $16(6)$ \\
\hline antOTS & $43(20)$ & $10(6)$ & $23(12)$ & $54(21)$ & $6(3)$ & $17(7)$ \\
\hline antMTG/ITG & $22(10)$ & $4(4)$ & $0(0)$ & $63(16)$ & $1(1)$ & $2(1)$ \\
\hline Total ATL & $\mathbf{1 2 1 ( 3 2 )}$ & $\mathbf{4 0 ( 2 0 )}$ & $\mathbf{5 7 ( 2 2 )}$ & $\mathbf{1 6 5 ( 2 9 )}$ & $\mathbf{5 1 ( 2 0 )}$ & $\mathbf{6 6}(19)$ \\
\hline
\end{tabular}




\section{Latero-medial spatial dissociation between face and house contacts}

Bayesian estimation was used for subsequent inferential analysis (e.g., Kruschke 2013). For each contrast we report the difference in central tendencies and a 95\% posterior high density confidence interval (HDI), which indicates the $95 \%$ most likely parameter values given the data (e.g., difference of mean face- and house amplitudes in latFG). For a given contrast, a $95 \% \mathrm{HDI}$ excluding a difference of 0 was interpreted as sufficient evidence for a difference (i.e., 0 was not among the $95 \%$ most likely values given the data), whereas a $95 \%$ HDI including a difference of 0 was interpreted as insufficient evidence for a difference (i.e., 0 was among the $95 \%$ most likely values given the data). See the Methods section for additional information on this approach.

Overall there was a larger proportion of significant face than house contacts $\left(p{ } p_{\text {diff }}=\right.$ $(537 / 3588)-(182 / 3588)=9.89 \%, 95 \% H D I=[8.52,11.27])$. Moreover, for faces, there was a higher proportion of significant contacts in the right as compared to the left hemisphere $\left(\right.$ prop $\left._{\text {diff }}=(314 / 1682)-(223 / 1906)=6.97 \%, 95 \% H D I=[4.61,9.31]\right)$. In contrast, for houses, there was an equal proportion of significant contacts in the right and the left hemispheres $\left(\right.$ prop $\left._{\text {diff }}=(82 / 1682)-(100 / 1906)=-0.37 \%, 95 \% H D I=[-1.8,1.09]\right)$. Finally, there was a larger proportion of face- than house- contacts in the OCC $\left(\right.$ prop $_{\text {diff }}=(91 / 381)-(33 / 381)=15.22 \%$, $95 \% H D I=[10.04,20.28]), \mathrm{PTL}\left(\operatorname{prop}_{\text {diff }}=(160 / 660)-(58 / 660)=15.45 \%, 95 \% \mathrm{HDI}=[11.51\right.$, $19.36])$, and the ATL $\left(\right.$ prop $\left._{\text {diff }}=(286 / 2547)-(91 / 2547)=7.65 \%, 95 \% H D I=[6.24,9.08]\right)$.

To visualize and quantify the proportions of face and house contacts at a group level, local proportions were computed and projected on the cortical surface (Figure 4 A). Consistent with previous work (e.g., Kadipasaoglu et al., 2016; Spiridon et al., 2006), we observed a medio-lateral spatial dissociation between house and face selective contacts. The 
lateral regions of the VOTC contained the largest proportion of face contacts, while the medial regions contained the largest proportion of house contacts (Figure 4A). This was confirmed statistically by comparing the percentage of house- and face-contacts along the $\mathrm{X}$ Talaraich axis (12 equally spaced bins with size $6 \mathrm{~mm}$ ), which was computed separately for each contact type by dividing the number of contacts in each bin by the total number of contacts for that specific contact-type across all bins and converting to percentage (Figure 5A; each curve sums to 100). This showed that the center of mass for house contacts $\left(\right.$ mean $\left._{t a l x}=28.39 \mathrm{~mm}\right)$ was more medial than that of face contacts $\left(\right.$ mean $_{\text {talx }}=38.79 \mathrm{~mm} ;$ mean $_{\text {diff }}=10.40 \mathrm{~mm}$, $95 \% H D I=[8.17,12.61] ;$ Figure 5A). 
A Proportion of face contacts

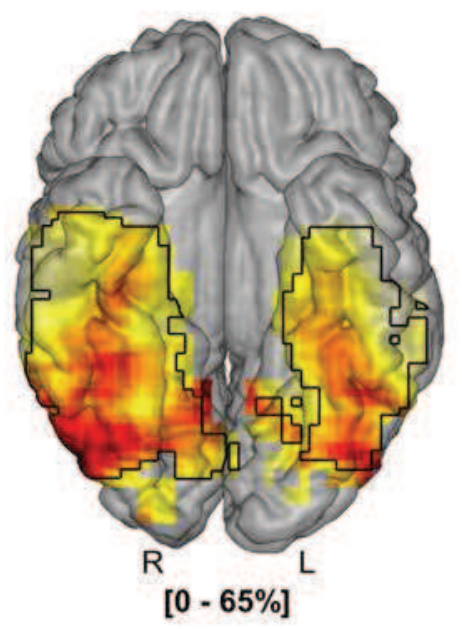

Proportion of house contacts

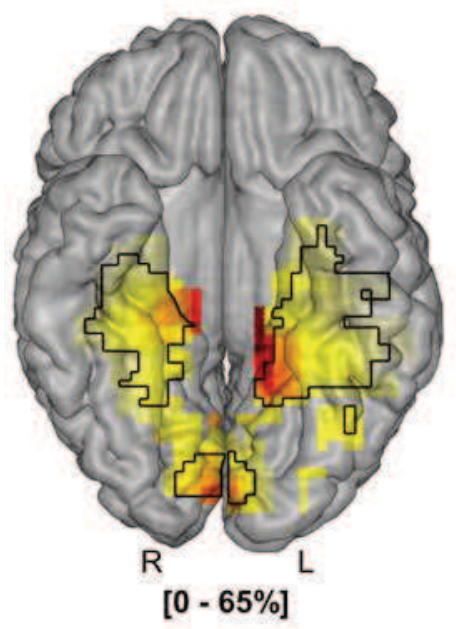

Proportion of overlap contacts

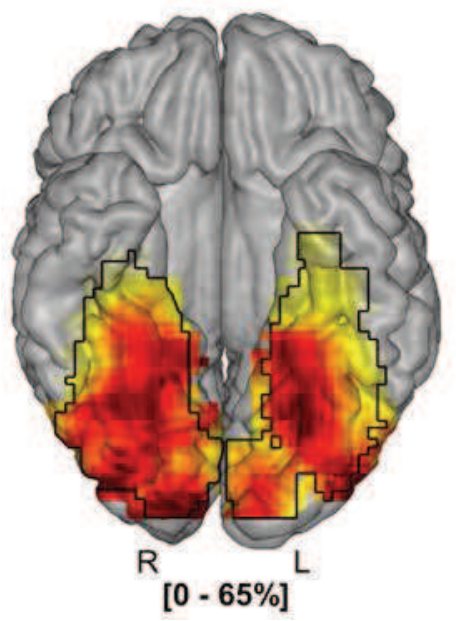

B Face-selective amplitude in face contacts

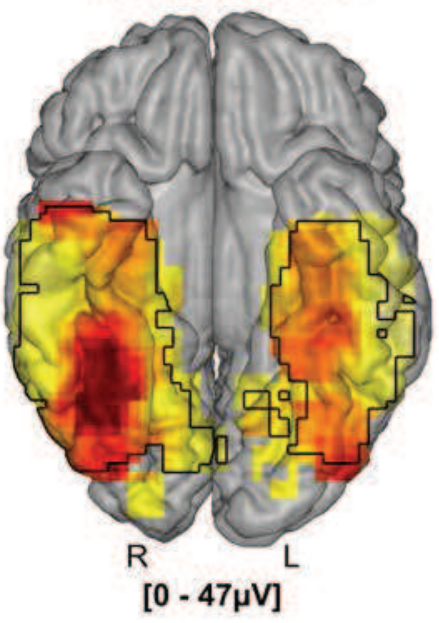

House-selective amplitude in house contacts

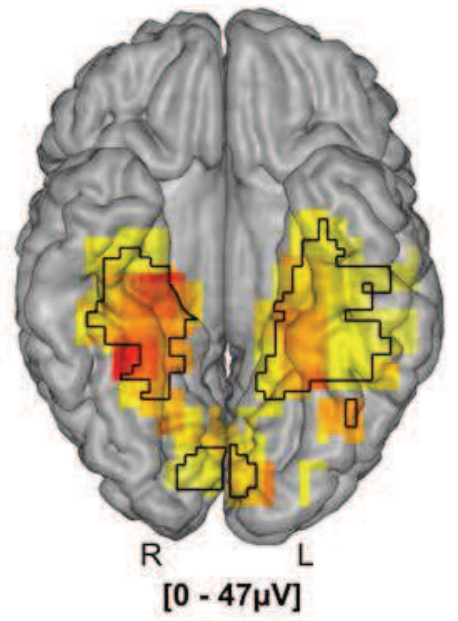

C Face-selective amplitude in overlap contacts

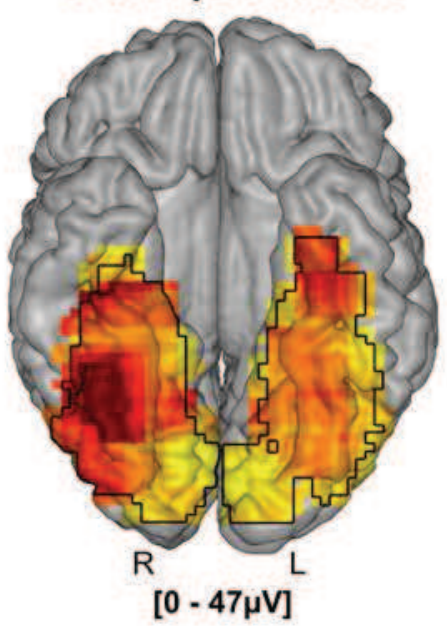

House-selective amplitude in overlap contacts

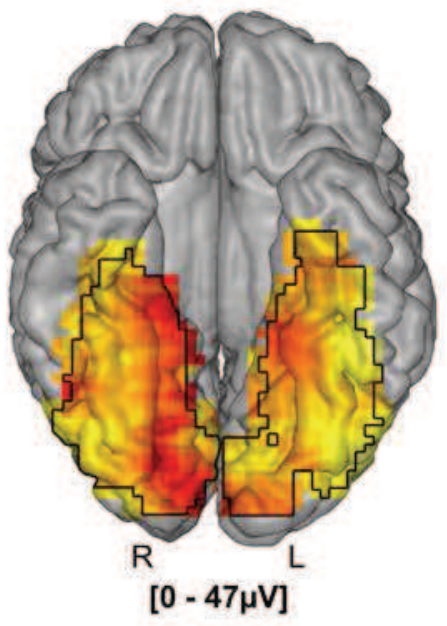

Max

Proportion (\%) I

Amplitude $(\mu \mathrm{V})$

Proportion $>0$

$(p<0.01)$ 
Figure 4. Proportion and amplitude maps. A. Maps of the local proportion of selective contacts relative to recorded contacts across VOTC for each contact type (face, house, overlap), displayed on the cortical surface. B. Maps of the local mean selective response amplitudes for face- and house-contacts. C. Maps of the local mean face- and houseselective response amplitudes in overlap contacts. Local proportions and amplitudes were computed in $15 \times 15$ voxels (for $X$ and $Y$ dimensions, respectively) using contacts collapsed over the Z dimension (superior-inferior) for better visualization. For the sake of replicability, only voxels containing significant responses from at least two individual brains were considered. Black contours outline proportions and amplitude significantly above zero. The letters below each hemisphere $(R, L)$ refers to the hemispheric side.
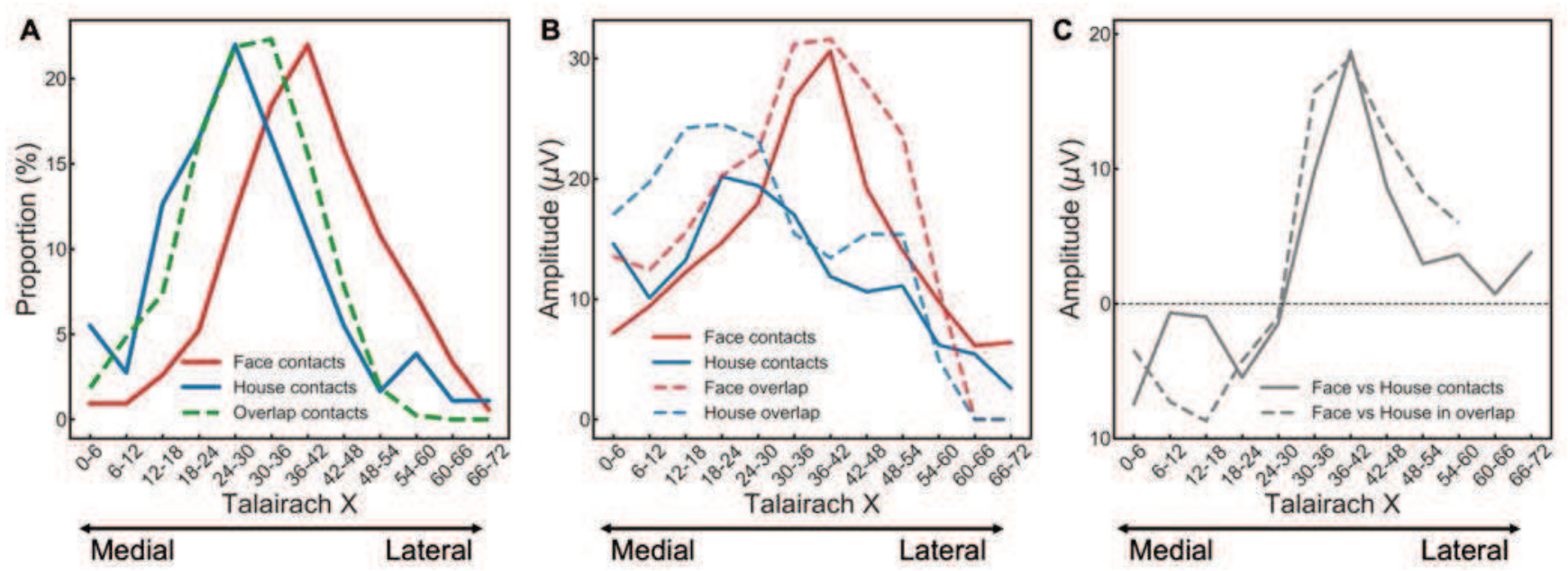

Figure 5. Medio-lateral organization to faces and houses across 6 equally spaced bins (width $=6 \mathrm{~mm}$ ) along the Talairach coordinates. A. Percentage of contacts as a function of Talairach coordinates and contact-type (hemispheres collapsed). The percentages were normalized by dividing the number of contacts in each bin by the total contacts across all bins separately for each contact-type, before converting to percentage (each curve sums to 100). 
B. Average selective response amplitudes to faces and houses as a function of $X$ Talairach in face and house contacts (solid lines) and overlap contacts (dashed lines). C. Face- and house-amplitude bias in face- and house-contacts (solid line) and overlap contacts (dashed line). Bias was computed by subtracting the average house-from average face-selective amplitude within each bin. Thus, amplitudes below and above 0 indicate house and face amplitude bias, respectively.

Next, we compared the relative proportion of face and house contacts within anatomical regions (e.g., latFG: face or house contacts / total of face and house contacts). A larger proportion of face than house contacts was observed in the IOG prop $_{\text {diff }}=(52 / 57)$ $(5 / 57)=82.46 \%, 95 \% H D I=[67.64,89.16])$, latFG $\left(\right.$ prop $_{\text {diff }}=(77 / 89)-(12 / 89)=73.03 \%$, $95 \% \mathrm{HDI}=[60.58,80.81]), \mathrm{MTG} / \mathrm{ITG}\left(\right.$ prop $_{\text {diff }}=(54 / 65)-(11 / 65)=66.15 \%, 95 \% \mathrm{HDI}=[50.35$, 76.13]), antFG ( prop $\left._{\text {diff }}=(15 / 19)-(4 / 19)=57.90 \%, 95 \% \mathrm{HDI}=[25.09,75.18]\right)$, antCOS $\left(\right.$ prop $\left._{\text {diff }}=(89 / 142)-(53 / 142)=25.35 \%, 95 \% H D I=[13.64,35.93]\right)$, antOTS $\left(\right.$ prop $_{\text {diff }}=$ $(97 / 113)-(16 / 113)=71.68 \%, 95 \% H D I=[60.70,78.94])$, and antMTG/ITG $\left(\right.$ prop $_{\text {diff }}=(85 / 90)$ $-(5 / 90)=88.89 \%, 95 \% H D I=[79.01,93.13] ;$ Figure $6 \mathrm{~A})$. In contrast, a larger proportion of house than face contacts were observed in the PHG $\left(\right.$ prop $_{\text {diff }}=(0 / 6)-(6 / 6)=-100 \%, 95 \% H D I$ $=[-96.64,-37.69])$ and the antPHG $\left(\right.$ prop $_{\text {diff }}=(0 / 13)-(13 / 13)=-100 \%, 95 \% H D I=[-98.27,-$ 65.03]). Finally, an equal proportion of face and house contacts was observed in the VMO $\left(\right.$ prop $\left._{\text {diff }}=(39 / 67)-(28 / 67)=16.42 \%, 95 \% \mathrm{HDI}=[-0.6,32.01]\right)$ and the medFG $\left(\right.$ prop $_{\text {diff }}=$ $(29 / 58)-(29 / 58)=0 \%, 95 \% H D I=[-17.66,17.67])$. As illustrated in Figure $6 \mathrm{~A}$, there was also a medial-to-lateral organization within OCC, PTL and ATL, as indicated by a decrease (or increase) in the proportion of house (or face) contacts moving from medial to lateral regions (logistic regression, where $b$ reflects a change in the odds of significant contacts moving from 
medial to lateral regions (negative reflects a decrease and increase in house- and facecontacts, respectively) : OCC: $b=-2.1,95 \% H D I=[-3.26,-1.09] ;$ PTL: $b=-1.11,95 \% H D I=[-$ $1.55,-0.69] ;$ ATL: $b=-0.85,95 \% H D I=[-1.1,-0.63])$. Thus, in terms of proportions, house and face contacts were organized across medial and lateral regions, respectively, and were found in both posterior and anterior parts of the VOTC. The anterior parts largely followed the patterns of the more posterior regions of the VOTC. 

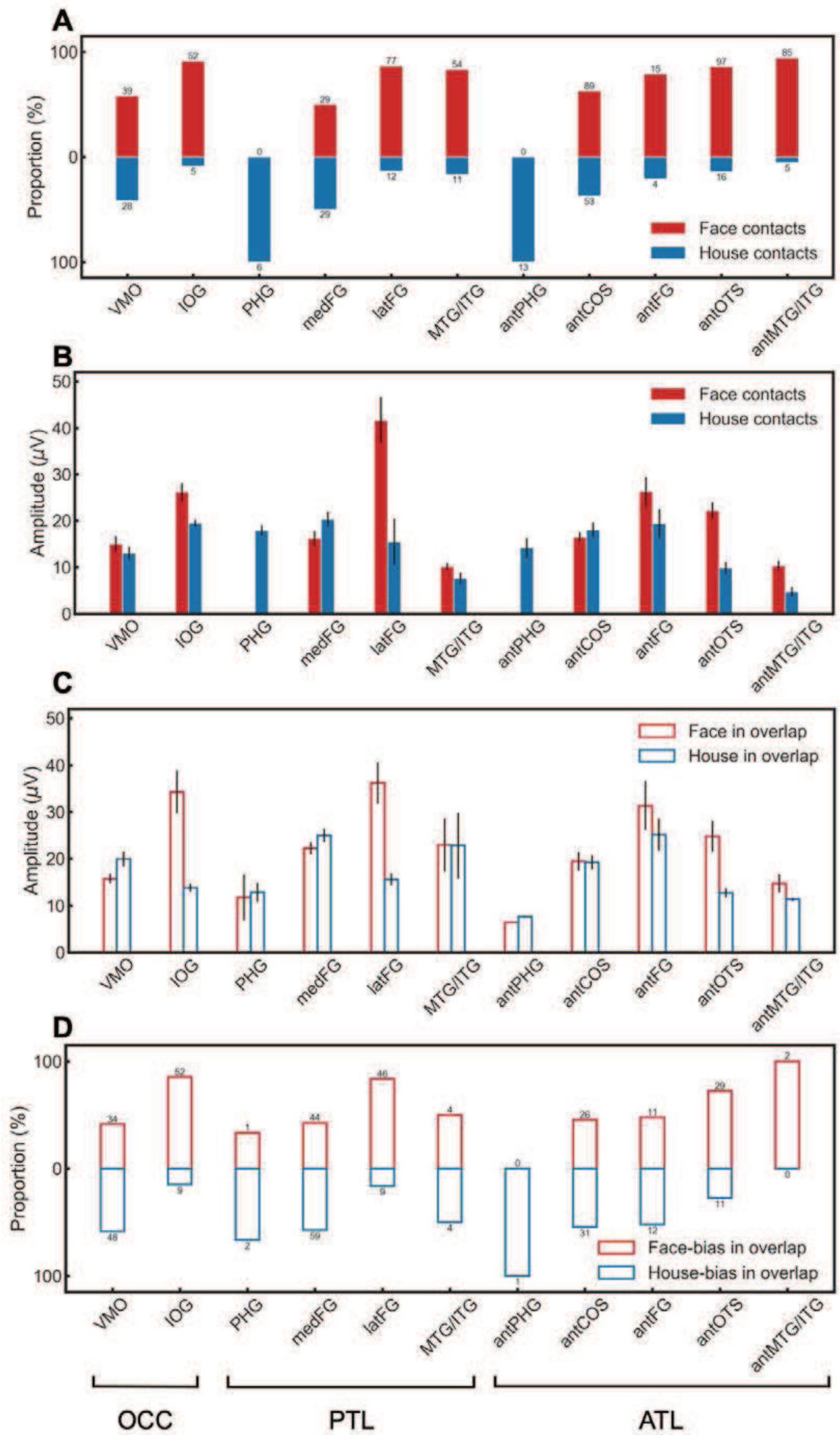

Anatomical Region 
Figure 6. Quantification of proportions and selective amplitudes in each anatomical region (hemispheres collapsed). A. Relative percentage of face and house contacts were computed in each region as the number of face (or house) contact out of the total of face and house contacts in that region. B. Selective response amplitudes were quantified in each region as the average of the selective response amplitudes across contacts, separately for face- and house-contacts. C. Selective response amplitudes were quantified in each region as the average of the response amplitudes across contacts, separately for face- and houseselective response amplitudes in overlap contacts. $\boldsymbol{D}$. Relative proportion of face and house biased overlap contacts were computed in each region as the number of face (or house) biased contact out of the total of overlap contacts in that region. Face and house bias were computed in each overlap contact by subtracting the house- from the face-selective response amplitudes. An overlap contact with positive value was categorized as a face-biased overlap contact, whereas one with a negative value was categorized as a house-biased overlap contact. Error bars represents SEMs. The number of significant contacts in each region is indicated by the number on top of each proportion bar.

We next quantified the selective response amplitudes recorded in face and house contacts. For each contact, we summed the baseline-subtracted amplitudes over harmonics of the $1.2 \mathrm{~Hz}$ face/house-selective response (from the $1 \mathrm{st}$ until the $14^{\text {th }}$, i.e., $1.2 \mathrm{~Hz}$ until 16.8 $\mathrm{Hz}$, excluding the 5th and 10th harmonics, i.e., $6 \mathrm{~Hz}$ and $12 \mathrm{~Hz}$ that coincided with the base frequency). Overall, the mean face-selective amplitude in face contacts was larger than the mean house-selective amplitude in house selective contacts $\left(\right.$ mean $_{\text {diff }}=20.60-15.56=5.04$ $\mu \mathrm{V}, 95 \% H D I=[2.62,7.48])$. Moreover, for both faces and houses, there was a higher selective amplitude in the right than the left hemisphere (Faces: mean $_{\text {diff }}=22.27-18.24=$ 
$4.03 \mu \mathrm{V}, 95 \% \mathrm{HDI}=[0.48,7.58] ;$ Houses: mean $_{\text {diff }}=17.49-13.97=3.52 \mu \mathrm{V}, 95 \% \mathrm{HDI}=[0.35$, 6.66]). These observations are consistent with recordings on the scalp (Jacques et al., 2016).

To visualize and quantify the selective amplitudes of face and house contacts at a finer spatial scale and group level, local average amplitudes were computed and projected on the cortical surface (Figure 4B). Amplitudes also showed a medio-lateral dissociation between house and face selective contacts (Figure 4B). This was confirmed statistically by first computing the mean selective-response amplitude within each X Talairach bin (12 equally spaced bins with size of $6 \mathrm{~mm}$ ) separately for each contact type (Figure 5B). Using the amplitude, for each contact type we generated a distribution of Talairach coordinates proportional to the mean selective amplitude in each bin. Specifically, for each X Talairach bin we generated a number of data points (with coordinates matching the center of the $X$ Talairach bin) that was directly proportional to the mean selective amplitudes in that bin (e.g., for amplitude $=2$ we generated 2 coordinate data points; for amplitude $=4$ we generated 4 coordinate data points; and so on). In the resulting distribution of $X$ Talairach coordinates across bins, the mode reflects the $\mathrm{X}$ Talairach coordinate with the highest amplitudes (unlike the mode for the count data, which reflects the $X$ Talairach coordinate with the highest density of contacts). Direct statistical comparison of the house- and face-scaled coordinate data, revealed that the house-selective amplitudes centered more medially $\left(\right.$ mean $_{\text {talx }}=29.66 \mathrm{~mm}$ ) than did the face-selective amplitudes $\left(\right.$ mean $_{\text {talx }}=35.14 \mathrm{~mm}$, mean $_{\text {diff }}=5.48,95 \% \mathrm{HDI}=[1.69$, 9.27]; Figure 5B). This medio-lateral-dissociation was also found in individual participants showing face- and house-selective responses in the same anatomical regions (higher faceselective responses in lateral regions and higher house-selective responses in medial regions, Figure S2). 
Next, we directly compared face and house amplitudes within anatomical regions. Maximum face-selective response amplitudes for face contacts were recorded in the latFG (Figure 6B), where, consistently with Jonas et al (2016), the response was larger in the right than the left hemisphere $\left(\right.$ mean $_{\text {diff }}=36.5 \mu \mathrm{V}, 95 \% H D I=[19.77,53.26]$, see Figure 4B top $)$. This difference was not found in proportion of face contacts (Figure 4A top), as analyzed by statistically comparing the proportion in right and left latFG voxels $(p<0.01$, permutation test). Direct comparisons between face- and house-selective responses amplitudes within each anatomical region (hemisphere collapsed, Figure 6B) showed that faces evoked larger response in the IOG $\left(\right.$ mean $\left._{\text {diff }}=6.73 \mu \mathrm{V}, 95 \% H D I=[1.64,11.76]\right)$, latFG $\left(\right.$ mean $_{\text {diff }}=26.22 \mu \mathrm{V}$, $95 \% \mathrm{HDI}=[10.51,41.95])$, antOTS $\left(\right.$ mean $\left._{\text {diff }}=12.37 \mu \mathrm{V}, 95 \% \mathrm{HDI}=[7.63,17.1]\right)$, and antMTG/ITG $\left(\right.$ mean $\left._{\text {diff }}=5.53 \mu \mathrm{V}, 95 \% H D I=[0.85,10.14]\right)$. In contrast, houses evoked larger amplitudes in the PHG $\left(\right.$ mean $\left._{\text {diff }}=-17.91 \mu \mathrm{V}, 95 \% H D I=[-21.72,-14.1]\right)$ and antPHG $\left(\right.$ mean $_{\text {diff }}$ $=-14.17 \mu \mathrm{V}, 95 \% \mathrm{HDI}=[9.03,19.24])$, since there were no face contacts in these regions (thus

relative to 0). Finally, houses and faces evoked equal amplitudes in the VMO $\left(\right.$ mean $_{\text {diff }}=$ $2.01 \mu \mathrm{V}, 95 \% \mathrm{HDI}=[-2.58,6.59]), \operatorname{medFG}\left(\right.$ mean $\left._{\text {diff }}=-4.15 \mu \mathrm{V}, 95 \% \mathrm{HDI}=[-8.99,0.69]\right)$, MTG/ITG $\left(\right.$ mean $\left._{\text {diff }}=2.48 \mu \mathrm{V}, 95 \% \mathrm{HDI}=[-1.05,6.0]\right)$, antCOS $\left(\right.$ mean $_{\text {diff }}=-1.65 \mu \mathrm{V}, 95 \% \mathrm{HDI}=$ $[-5.53,2.24])$, and antFG $\left(m^{2} a_{\text {diff }}=6.92 \mu \mathrm{V}, 95 \% \mathrm{HDI}=[-13.52,26.89]\right)$. Overall, the amplitude patterns were largely consistent with the patterns observed in the proportion data.

\section{Spatial dissociation between face and house contacts is maintained in the ATL}

We examined whether the well-known face-house spatial dissociations extend into the ATL, largely affected by a magnetic artefact in fMRI. Figure 7A shows the distribution of face and house contacts in the ATL as a function of $\mathrm{X}$ Talairach (medio-lateral) and $\mathrm{Y}$ Talairach (postero-anterior) axes, where the color of the line indicate the density of the respective contacts (face and house densities are relative to itself). A medio-lateral dissociation was 
confirmed statistically in the ATL by comparing mean X Talairach coordinates for face- and house-contacts, which showed that the center of mass for house contacts (mean $_{\text {talx }}=31.42$ $\mathrm{mm})$ was more medial than that of face contacts $\left(\right.$ mean $_{\text {talx }}=40.34 \mathrm{~mm} ;$ mean $_{\text {diff }}=8.92 \mathrm{~mm}$, $95 \% \mathrm{HDI}=[6.36,11.45]$; Figure $7 \mathrm{~A})$, which is in agreement with our proportion and amplitude analyses across ATL anatomical regions (see above). Moreover, a comparison of the mean $Y$ Talairach (postero-anterior) coordinates for face- and house-contacts showed that the center of mass for house contacts $\left(\right.$ mean $\left._{\text {taly }}=-23.11 \mathrm{~mm}\right)$ was more posterior than that of face contacts $\left(\right.$ mean $_{\text {taly }}=-18.80 \mathrm{~mm} ;$ mean $_{\text {diff }}=-4.31 \mathrm{~mm}, 95 \% \mathrm{HDI}=[1.94,6.67]$; Figure 7A).

Next, we examined the medio-lateral and postero-anterior dissociation between houses and faces in specific anatomical regions of the ATL. To do so, we combined the Talairach coordinates and the anatomical locations of face and house contacts in the ATL. We considered the $\mathrm{X}$ coordinates in the different ATL anatomical regions to examine in which region the medio-lateral transition of the ATL is likely to occur (Figure 7B). Only the antCOS, containing both numerous face and house contacts, showed a medio-lateral dissociation, with the central mass of houses $\left(\right.$ mean $_{\text {talx }}=28.26 \mathrm{~mm}$ ) being more medial than the central mass of faces $\left(\right.$ mean $_{\text {talx }}=30.47 \mathrm{~mm} ;$ mean $_{\text {diff }}=2.21 \mathrm{~mm}, 95 \% \mathrm{HDI}=[0.3,4.13] ;$ Figure $\left.7 \mathrm{~A}\right)$. No other regions in the ATL contained a medio-lateral dissociation (all 95\%HDls included 0 ). This shows that the antCOS is an anatomical landmark between face- and house selective regions in the ATL. Second, we examined posterior-anterior dissociations within regions of the ATL (Y coordinates of face and house contacts in the different ATL anatomical regions, Figure 7C). Again, a dissociation was found only in the antCOS, where the central mass of houses $\left(\right.$ mean $\left._{\text {taly }}=-23.02 \mathrm{~mm}\right)$ was more posterior than that of face contacts $\left(\right.$ mean $_{\text {taly }}=-19.03 \mathrm{~mm}$; mean $_{\text {diff }}=3.99 \mathrm{~mm}, 95 \% \mathrm{HDI}=[0.56,7.38] ;$ Figure $\left.7 \mathrm{~A}\right)$. This dissociation was not found in any other regions (all 95\%HDls included 0). The antPHG, containing only house contacts, did not 
show very anterior house contacts (except one, Figure 7C). As it is shown in Figure 7C, while face-selective contacts are distributed all along the postero-anterior axis of the antMTG/ITG, antOTS, antFG and antCOS, the house contacts are clustered in the posterior parts of the (medial) antCOS and the antPHG (Figure 7A and 7C).
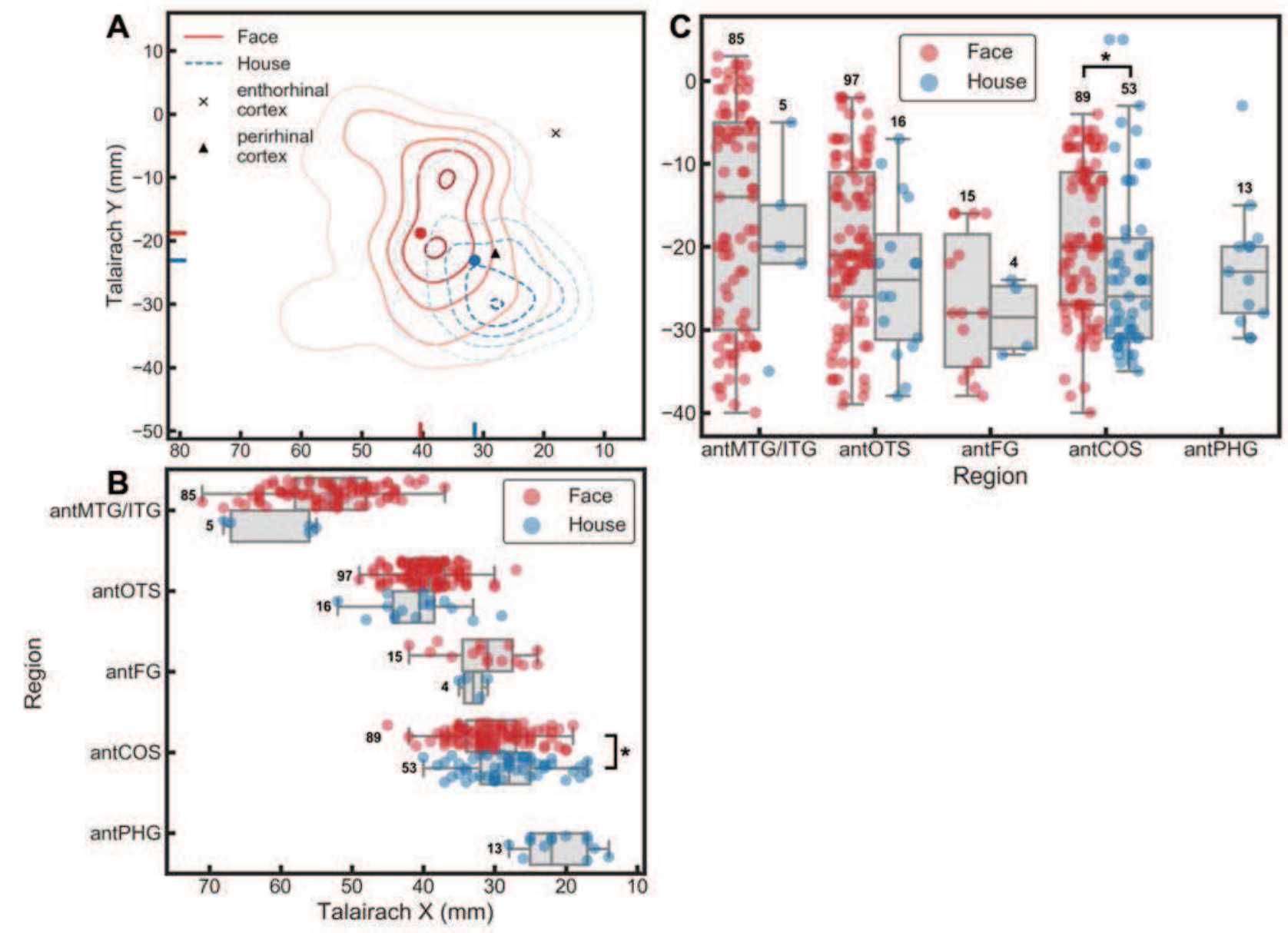

Figure 7. Spatial dissociation between face and house contacts in the ATL. A. Contour plot showing distribution of face and house contacts along the medio-lateral (x-axis) and postero-lateral axis (y-axis). Each distribution is computed relative to itself and darker contour colors indicate larger density of contacts. The central mass for each distribution is plotted as a solid dot within the contour plot and as a vertical line on the $x$ - and $y$-axis. The central mass 
coordinates of the entorhinal and perirhinal cortices extracted from the Brede Database are plotted within the figure. B. $X$ coordinates of face and house contacts in the different ATL anatomical regions. The boxplot displays three quartiles (Q1, median, Q2) and the whiskers extend to points that lie within 1.5 interquartile range (IQRs) of the lower and upper quartile. * indicates that the 95\% HDI did not include 0 . The number next to the error bar indicate the number of contacts. Note that the strength of the color of contacts (relative to legend) indicates the contact-density along the Talairach X. C. Y coordinates of face and house contacts in the different ATL anatomical regions. Same convention as for panel B.

\section{Dissociations between face and house amplitudes in overlap contacts.}

A large portion of contacts responded to both faces and houses (overlap contacts, Figure 3). They were equally distributed in both hemispheres prop diff $=(213 / 1906)-$ $(222 / 1682)=-2.02 \%, 95 \% H D I=[-0.114 .17])$ and local proportion maps showed a wide distribution across OCC and PTL (without a clear medial or lateral bias) while density was low in the ATL (Figure 4A). We first characterize the spatial location of overlap contacts along the X Talairach axis (medial - lateral). The statistical comparison of the normalized percentages of overlap contacts with that of faces and houses (the contact-count in each bin divided by the sum within contact-type and converted to percentage) showed that the central mass of overlap contacts $\left(\right.$ mean $\left._{\text {talx }}=28.96 \mathrm{~mm}\right)$ differed from that of face $\left(\right.$ mean $_{\text {diff }}=9.83 \mathrm{~mm} ; \mathrm{HDI}=$ $[8.43,11.26])$, but not house contacts $\left(\right.$ mean $_{\text {diff }}=-0.57 \mathrm{~mm} ; \mathrm{HDI}=[-2.78,1.62]$; Figure $\left.5 \mathrm{~A}\right)$.

What do the overlap contacts represent? Since these contacts are selective to both faces and houses, they could reflect domain-general responses that originate in mechanisms that discriminate both face and house stimuli (or a repeating category among less frequently repeated categories). According to this account, overlap contacts should have similar 
response profiles to both face- and house-stimuli. Alternatively, the overlap contacts could reflect dissociated face and house selective responses originating from distinct neural sources close to the same contact. In this latter scenario, one would expect faces and houses to evoke dissociated responses (e.g., spatially dissociated maximum amplitudes; noncorrelated amplitudes). To disentangle these accounts, we quantified and compared in each overlap contact the selective responses evoked separately by faces and houses (sum over harmonics, as for face and house contacts and computed local amplitude maps).

We first examined their amplitudes along the $X$ Talairach axis. Notably, the house and face amplitude profiles of the overlap contacts mirrored the profiles measured for house and face contacts in two ways. First, following the same approach as with house- and facecontacts (generating the $\mathrm{x}$-coordinates proportional to its mean amplitude, see above) revealed that the house-selective amplitudes centered more medially $\left(\right.$ mean $\left._{\text {talx }}=25.95 \mathrm{~mm}\right)$ than did the face-selective amplitudes $\left(\operatorname{mean}_{\text {talx }}=32.31 \mathrm{~mm}\right.$, mean $_{\text {diff }}=6.36 \mathrm{~mm}, 95 \% \mathrm{HDI}=$ [3.29, 9.50]; Figure 5B). Second, within each bin, we subtracted the mean amplitude between house- and face-responses (dashed line) within the overlap contacts, as well as between house- and face-contacts (solid line) (Figure 5C). There was a strong correlation between the bias reflected by the solid and dashed lines $(r=0.87, n=12,95 \% H D I=[0.48,0.99]$; $\mathrm{X}$ Talairach bins with no data (i.e., 60-66, 66-72 for overlap) was set to 0 to indicate neither face nor house bias). For both analyses, we found similar results with house biased amplitudes centered more medially and face biased amplitudes more laterally. Such similarity is consistent with the claim that the house and face sources measured by the overlap contacts originate medially and laterally, respectively.

Second, we computed maps separately for face and house selective- response amplitudes in overlap contacts (Figure 4C). At local levels, faces evoked maximum amplitude 
in the same lateral regions (especially the right IOG and right latFG) as in face contacts, whereas houses evoked maximum amplitude in some of the same medial regions as in house contacts (Figure 4C). Direct comparisons between face and house response amplitudes within each anatomical region showed that faces evoked larger responses than houses in the IOG $\left(\right.$ mean $\left._{\text {diff }}=20.51 \mu \mathrm{m}, 95 \% \mathrm{HDI}=[11.03,30.03]\right)$, latFG $\left(\right.$ mean $_{\text {diff }}=20.62 \mu \mathrm{m}, 95 \% \mathrm{HDI}=$ $[11.73,29.55])$, and the antOTS $\left(\right.$ mean $_{\text {diff }}=12.07 \mu \mathrm{m}, 95 \% H D I=[4.65,19.47] ;$ Figure $\left.6 \mathrm{C}\right)$. In contrast, houses evoked larger response amplitudes than faces in the VMO $\left(m^{2} a_{\text {diff }}=\right.$ $4.21 \mu \mathrm{m}, 95 \% H D I=[-7.59,-0.82])$. Equal amplitudes were found in the medFG $\left(m^{2} n_{\text {diff }}=-\right.$ $2.74 \mu \mathrm{m}, 95 \% H D I=[-5.87,0.39]), \mathrm{MTG} / \mathrm{ITG}\left(\operatorname{mean}_{\text {diff }}=0.09 \mu \mathrm{m}, 95 \% H D I=[-11.84,12.13]\right)$, antCOS $\left(\right.$ mean $\left._{\text {diff }}=0.22 \mu \mathrm{m}, 95 \% \mathrm{HDI}=[-3.78,4.24]\right)$, and antFG $\left(\right.$ mean $_{\text {diff }}=6.20 \mu \mathrm{m}, 95 \% \mathrm{HDI}$ $=[-7.56,19.97])$. Finally, there were too few contacts to estimate the mean amplitudes in the PHG $(n=3)$, antPHG $(n=1)$, and antMTG/ITG $(n=2)$. Collectively, these patterns largely mirrored that of the face and house contacts (compare Figure 4B and C; compare Figure 6B and C).

Third, we computed the proportion of face-biased contacts (selective-response amplitude face > house) and house-biased contacts (selective-response amplitude house > face) among all overlap contacts across regions (Figure 6D). We compared the relative proportion of face and house biased contacts within local regions (e.g., latFG: face- or housebiased contacts/total of overlap contacts). A larger proportion of face than house biased contacts was observed in the IOG $\left(\right.$ prop $_{\text {diff }}=(52 / 61)-(9 / 61)=70.49 \%, 95 \% \mathrm{HDI}=[54.62$, 79.83]), latFG $($ prop diff $=(46 / 55)-(9 / 55)=67.27 \%, 95 \% H D I=[50.03,77.63])$, and antOTS $\left(\right.$ prop $_{\text {diff }}=(29 / 40)-(11 / 40)=45 \%, 95 \% H D I=[22.91,61.04] ;$ Figure 6D $)$. In contrast, a larger proportion of house than face contacts were observed in the VMO (prop diff $=(34 / 82)-(48 / 82)$ $=-17.07 \%, 95 \% H D I=[-31.34,-1.67])$ and medFG $\left(\right.$ prop $_{\text {diff }}=(44 / 103)-(59 / 103)=-14.56 \%$, 
$95 \% H D I=[-27.53,-0.73])$. An equal proportion of face and house contacts was observed in the MTG/ITG $\left(\right.$ prop $\left._{\text {diff }}=(4 / 8)-(4 / 8)=0 \%, 95 \% H D I=[-41.32,41.35]\right)$, antCOS $\left(\right.$ prop $_{\text {diff }}=$ $(26 / 57)-(31 / 57)=-8.77 \%, 95 \% H D I=[-26.17,9.43])$, and antFG $($ prop diff $=(11 / 23)-(12 / 23)$ $=-4.35 \%, H D I=[-30.93,23.36])$. Finally, there were too few contacts to estimate the relative proportions in the PHG $(n=3)$, antPHG $(n=1)$, and antMTG/ITG $(n=2)$. Importantly, across regions, the proportions of face- and house biased contacts correlated strongly with the respective proportions of face and house contacts (compare Figure $6 \mathrm{~A}$ and $\mathrm{D} ; r=.87, n=11$, $95 \% H D I=[0.48,0.99])$.

Finally, we correlated face and house selective-response amplitudes computed at the individual contact level. Across all overlap contacts, the face- and house-selective response amplitudes $(1.2 \mathrm{~Hz}$ and harmonics) correlated weakly $(r=0.16, n=435,95 \% \mathrm{HDI}=[0.07$, 0.25]; Figure $8 \mathrm{~A}$ ), despite that the exact same contacts showed an almost perfect correlation to the base responses (i.e., $6 \mathrm{~Hz}$ and harmonics; $r=0.98, n=435,95 \% \mathrm{HDI}=[0.96,1]$, Figure $8 \mathrm{C}$ ). This suggests that while the base response was largely generated by the same neural populations, the selective response at the same contacts was generated largely by dissociated neural populations. Even after removing face- and house-selective amplitudes higher than $z=3$ ( $z$ score computed separately, 18 "outlier" contacts removed), the correlation of category-selective response remained weak (i.e., $r=0.26, n=417,95 \% \mathrm{HDI}=$ $[0.17,0.36]$, Figure $8 \mathrm{~B}$ ) and substantially lower than the correlation for base responses.

Taken together, these results showed that overlap contacts reflected to a large extent dissociated face- and house-selective responses that are both captured by a common contact, typically located between face- and house-selective regions. 

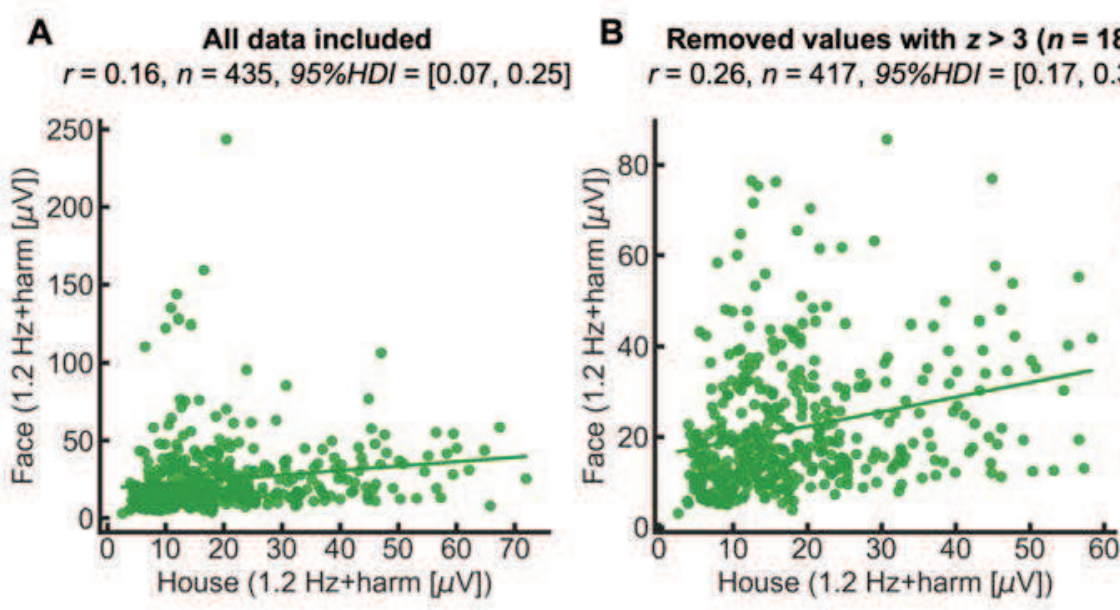

C All data included $r=0.96, n=435,95 \% H D I=[0.96,1]$

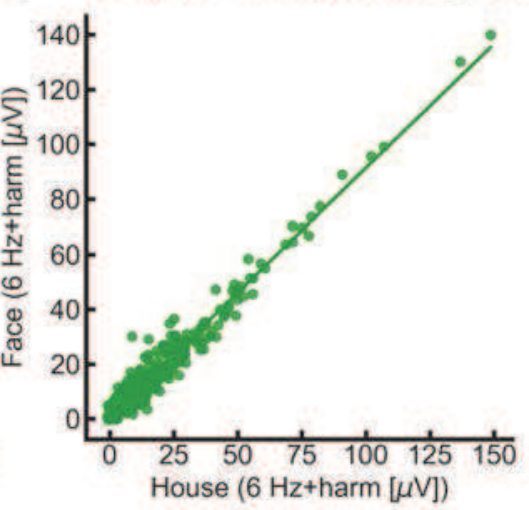

Figure 8. Amplitude correlations with overlap contacts. A. Correlation between the face(y-axis) and house- (x-axis) selective response amplitudes within the overlap contacts. $\boldsymbol{B}$. Correlation between the face- (y-axis) and house- (x-axis) selective response amplitudes within the overlap contacts, after removing contacts with a selective amplitude higher than $z=$ 3 (18 contacts removed). C. Correlation between the base response amplitudes in the face sequences (y-axis) and the base response amplitudes in the house sequences (x-axis) within the overlap contacts.

\section{Category-exclusive responses}

A previous SEEG-FPVS study (Jonas et al. 2016) found contacts in the ATL that exhibited exclusive responses to faces, i.e., significant face responses without any significant general visual response to nonface objects at the base frequency. However, due to their anterior location, it could be speculated that these responses (rather than being face exclusive) reflected a domain-general response to a predictable event (oddball faces) among non-predictable events (base objects). If the latter account is correct, then the face-exclusive contacts should also be selective to houses (i.e., overlap contacts with similar selective 
responses for both categories). To detect exclusive contacts in our sample, we assessed the significance of the base response $(6 \mathrm{~Hz}$ and harmonics) on each selective contacts (face, house, overlap) using the same method as for the discrimination responses (epoching the FFT spectrum into segments centered at the base frequency and harmonics, from the 1st until the 4th, i.e., $6 \mathrm{~Hz}$ until $24 \mathrm{~Hz}$, summing the 4 FFT segments, transforming the summed FFT spectrum into a Z-score). A selective contact was considered as exclusive if the base response was non-significant in both the face and the house conditions $(Z$-score $<3.1)$.

In total, we found 225 exclusive contacts $(19.50 \%$ of significant contacts; $6.34 \%$ of total recorded; 59 unique patients). Consistent with Jonas et al. (2016), most of these contacts were located in the ATL $(160 / 225=71.11 \%)$. We found exclusive contacts specific to one category (face-exclusive $=160$; house-exclusive $=41$ ) as well as exclusive contacts responsive to both categories (overlap-exclusive $=24$ ). An example of each contact is shown in Figure 9. Most of exclusive contacts were specific to one category $([160+41] / 225=$ $89.33 \%$ ). Out of the total number of exclusive contacts, there was a much larger proportion of face-exclusive than house-exclusive contacts $\left(\right.$ prop $_{\text {diff }}=(160 / 225)-(41 / 225)=52.89 \%$, $95 \% H D I=[44.44,59.98])$, and a larger proportion of house-exclusive than overlap-exclusive contacts $\left(\right.$ prop $\left._{\text {diff }}=(41 / 225)-(24 / 225)=7.56 \%, 95 \% H D I=[1.05,14.01]\right)$. Out of the number of total selective contacts within each contact type (e.g., face, house and overlap contact), there was no difference in the proportion of face and house exclusive contacts $\left(\right.$ rrop $_{\text {diff }}=$ $(160 / 537)-(41 / 182)=7.27 \%, 95 \% H D I=[-0.31,14.03])$. However, the proportions of faceand house-exclusive contacts were larger than the proportion of overlap contacts (face vs overlap: prop $_{\text {diff }}=(160 / 537)-(24 / 435)=24.28 \%, 95 \% H D I=[19.74,28.56]$; house vs overlap: prop $\left._{\text {diff }}=(41 / 182)-(24 / 435)=17.01 \%, 95 \% H D I=[10.94,23.74]\right)$. The spatial distribution of each exclusive contact type (face, house, overlap) at group level is shown in Figure 9. 
Thus, the finding of mostly category-exclusive contacts contradicts the claim that the exclusive contacts reflect domain-general responses to a predictable event, but rather suggest a change in response profile of category-specific (e.g., face, house) processes towards anterior regions. 


\section{Group maps}
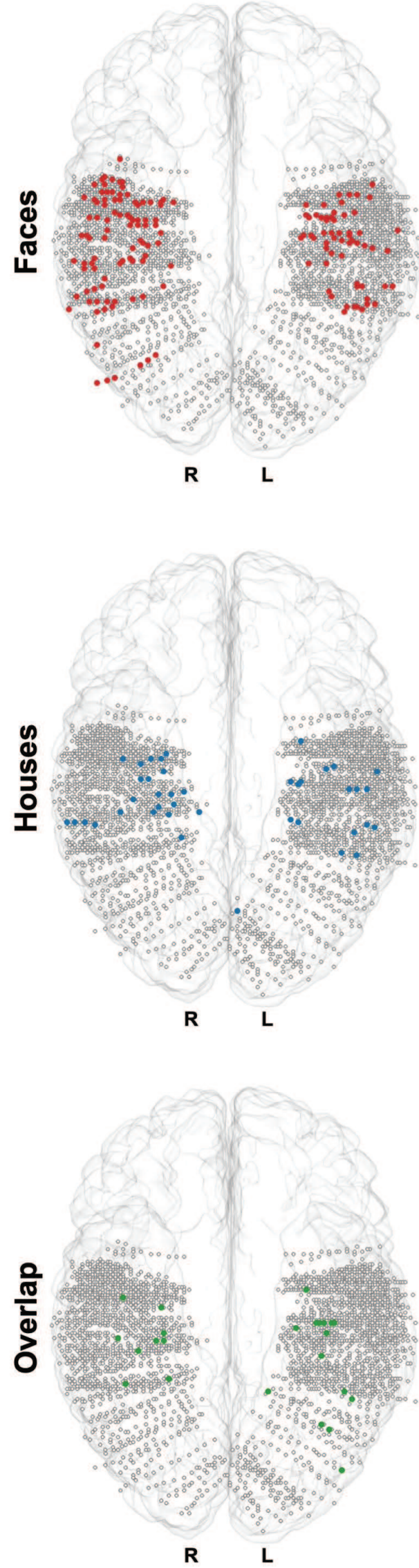

Sample contacts
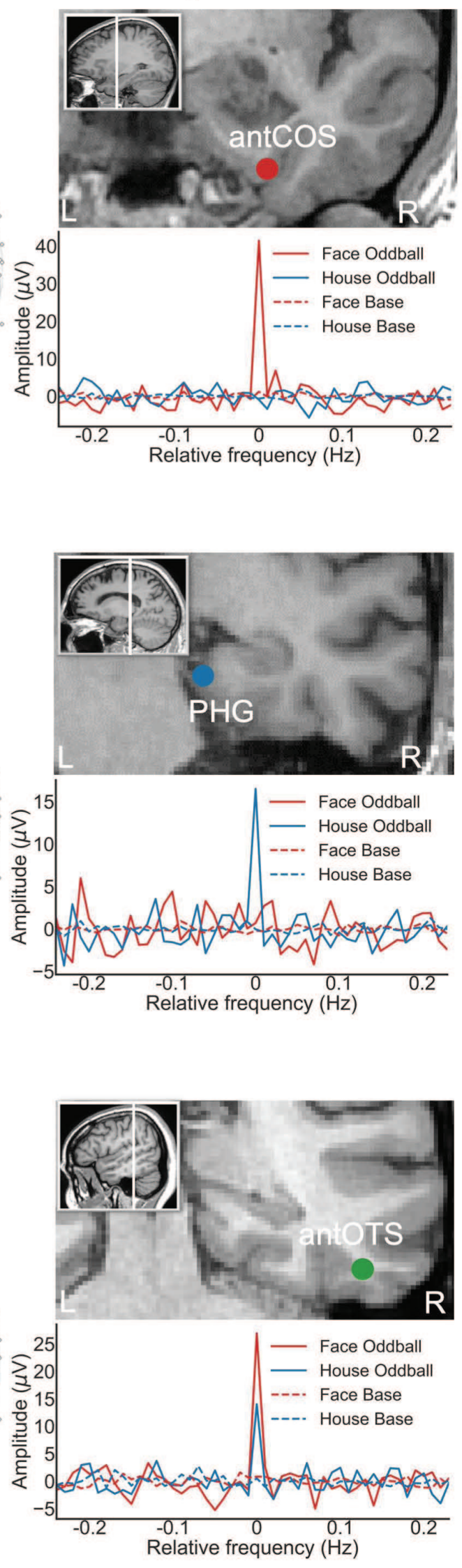
Figure 9. Classification and distribution of three types of exclusive discrimination contacts. Left. Maps of all 3588 VOTC recording contacts across the 75 individual brains displayed in the MNI space using a transparent reconstructed cortical surface of the Colin27 brain. Each circle represents a single contact. Color filled circles correspond to exclusive contacts colored according to their category (face, house, overlap). These were contacts that showed a significant selective response (to faces only, houses only or both), but no significant response to other objects at the base frequency. White-filled circles correspond to contacts on which no exclusive responses were recorded. Right. Examples of summed FFT spectra showing both selective and base responses for each contact type (3 individual contacts in 3 different participants). Top: face-exclusive contact, selective to faces only with no base responses, middle: house-exclusive contact selective to houses only with no base responses; bottom: overlap-exclusive contact selective to both faces and houses with no base responses. Their anatomical location is illustrated in the respective coronal MRI slices.

\section{Discussion}

\section{The functional specificity of the FPVS-SEEG approach}

Testing 28 patients with the same paradigm as used here but with faces as the only category of interest, Jonas et al. (2016) reported clusters of high-amplitude face-selective contacts with a peak in the right latFG, supporting the dominant role of this region in face categorization as identified previously with fMRI (e.g., Kanwisher et al., 1997). They also reported a wide distribution of face-selective responses across the VOTC, including the underexplored ATL. These findings are largely replicated here with a much larger group of patients $(N=75)$, providing a much denser sampling of the VOTC (3588 contacts in the grey matter in the present study compared to 1678 contacts in Jonas et al. 2016). Most 
importantly, the present study goes well beyond previous evidence by showing that the wide distribution of face-selective responses in the human VOTC does not reflect a lack of specificity of the FPVS-SEEG approach (i.e., responses to any highly familiar category appearing periodically in a continuous stream of various non-face objects and/or responses from very distant sources), for several reasons. First, although face- and house-selective responses are found in the VOTC, they elicit largely spatially distinct VOTC maps, both in amplitude and proportion of significant contacts, in lateral regions for faces and in medial regions for houses. This observation is consistent with observations of fMRI studies that have directly contrasted the two categories (e.g., Weiner and Grill-Spector 2010, Spiridon et al. 2006, Nasr et al. 2011, Tootell et al. 2008, Gomez et al. 2015) but also with the findings of an intracranial (Electrocorticography, ECoG) study focusing on more restricted regions and with smaller sample tested (Kadipasaoglu et al. 2016). Second, most (62.4\%) of the significant contacts were selective to one category only, either faces or houses. Third, the wide distribution of face-selective responses was still present when considering only the contacts selective to faces and not to houses. Fourth, there was a higher proportion of significant face than house contacts and a higher amplitude recorded on face contacts (consistently with observations made on the scalp on neurotypical observers tested with the same approach, Jacques et al. 2016), as well as a specifically higher proportion in the right than in the left hemisphere only for face contacts. This last result is in agreement with the long-standing observation of a right hemispheric dominance for faces (Meadows, 1974; see Rossion 2014). Altogether, these observations show that selective responses recorded with the SEEG-FPVS approach are specific to the tested category and not merely due or related to the periodic repetition of any category in a continuous stream of various nonface objects (Quek and Rossion 2017).

Spatial dissociation between face- and house-selective responses extends to the ATL 
Previous fMRI and intracranial EEG studies comparing face- and house selective responses found a medio-lateral dissociation in the posterior VOTC only (fMRI: Spiridon et al. 2006; Nasr et al. 2011; intracranial EEG: Kadipasaoglu et al. 2016; Jacques et al. 2016). A recent fMRI study showed that place-selective voxels in the medial VOTC are not found anteriorly to the anterior tip of the mid-fusiform sulcus, i.e., not anteriorly to the latFG and medFG (Weiner et al., 2018). Here we found that this spatial dissociation extends to the ATL, mostly because house-selectivity extends into the antPHG and antCOS (at least in their posterior parts, as indicated by the group maps; Figures 3 and 4). By combining the individual anatomy and the Talairach coordinates, we found a medio-lateral dissociation within the antCOS, showing that this sulcus is an anatomical landmark between face- and houseselective regions in the ATL (houses: antPHG and medial part of the antCOS; faces: lateral part of the antCOS, antOTS, antFG, antMTG/ITG). Within the antCOS, we also found a postero-lateral dissociation, with house contacts being clustered in the posterior part of the antCOS. Moreover, in the antPHG, containing only house contacts, these contacts are not located very anteriorly. These observations are probably explained by the fact that the anterior parts of the (medial) antCOS and antPHG are known to be the location the perirhinal cortex and entorhinal cortex respectively (Van Hoesen, 1995, Van Hoesen et al., 2000), both belonging to the medial temporal lobe (MTL) memory system. This suggests that, along the postero-anterior axis of the (medial) antCOS and antPHG, these is a transition from a highlevel visual area (house-selective) to the MTL memory system involved in episodic memory. These results also help clarifying the relationship between the face-selective responses found in the antCOS belonging to the cortical face network (the present data, Jonas et al., 2016, Tanji et al., 2012, Rajimehr et al., 2009, Rossion et al., 2012, Tsao et al., 2008) and the perirhinal cortex involved in episodic memory (e.g., see Collins and Olson, 2014). The present data suggest that these regions are spatially segregated: while the face-selective responses 
are located all along the lateral part of the antCOS, the perirhinal cortex is restricted to the medial and anterior part of the antCOS.

This spatial dissociation in the ATL has not been found in fMRI studies, probably because the signal drop-out affects both face- and house-selective responses in this region (e.g., Spiridon et al. 2006; Nasr et al. 2011). Intracranial EEG studies using ECoG with a high SNR in the ATL also failed to find such dissociation in anterior regions of the temporal lobe (Kadipasaoglu et al. 2016; Jacques et al. 2016). For example, despite a wide sampling across the VOTC, Kadipasaoglu et al. (2016) did not report category-selective responses anteriorly to the anterior tip of the middle fusiform sulci, in the ATL (for faces or houses). This could be because ECoG electrodes as used by Kadipasaoglu et al. (2016) are restricted to the gyral surface and may therefore be less sensitive to ATL category-selective responses located mainly in sulci, as captured here with the SEEG approach.

\section{The overlap between face-selective and house-selective responses}

In the current FPVS paradigm, responses to face stimulation measured at $1.2 \mathrm{~Hz}$ and harmonics are face-selective responses, i.e. responses that reflect a contrast with nonface objects presented in the FPVS sequence. This contrast response is generalized across widely variable exemplars, providing a generic face categorization response in the human brain (see Rossion et al. 2018). However, interestingly, a proportion of contacts that measured a selective response to faces also recorded significant selective responses to houses. These responses could either (1) reflect domain-general processes that originate in populations of neurons that discriminate any repeated category among other categories, or (2) reflect functionally distinct populations of neurons whose responses are measured at the same recording contacts. Consistent with the latter account, the low correlation between the amplitude of face- and house-selective responses in the overlap contacts provides no evidence that these responses originate from the same domain-general underlying process. 
Moreover, the spatial dissociation between the response amplitudes to faces and houses in overlap contacts closely matched that of face and house contacts (Figure $4 ; 5$ ), providing further evidence that the overlap contacts reflect dissociated face- and house-selective responses.

In the case of dissociated face- and house-selective responses projecting to the same contact, two main scenarios could be considered. First, category-selectivity could gradually decrease, so that adjacent category-selective populations of neurons could be partly intermingled (Spiridon et al. 2006; Kadipasaoglu et al. 2016). Second, face- and houseselective neurons could be located in spatially distinct but nearby anatomical regions, and some recording contacts may measure responses generated by both regions due to volume conduction of the electrical field (Herreras 2016; Buzsáki et al. 2012). Current fMRI findings on spatial organization of category-selectivity in VOTC is compatible with both scenarios, with evidence for spatially separated face-selective and house-selective regions but with potentially graded selectivity along the medio-lateral axis (Tootell et al. 2008; Spiridon et al. 2006). It is important to note that some fMRI studies may have artificially increased the spatial dissociation between face- and house-selective regions since they directly contrasted faces and house stimuli (e.g., Nasr et al. 2011; Tootell et al. 2008). Further investigation will be needed to disentangle the different scenarios.

How local electrophysiological activities recorded with intracranial electrodes (using SEEG or ECoG) are is a matter of debate (e.g., Zaveri et al. 2009; Wennberg 2010; Dubey and Ray 2019; Yoshor et al. 2007). This issue is not specific to the present FPVS approach and concerns intracranial EEG studies in general. Here, according to one of the scenarios outlined above, some responses could be related to volume conduction from distant source. For example, a recent ECOG study in the monkey visual cortex showed that the spatial spread was around $3 \mathrm{~mm}$ (Dubey and Ray 2019). However, despite this uncertainty, the 
FPVS-SEEG approach as used here is able to provide distinct and valid category-selectivity maps across the whole VOTC, even if different sources could project to the same electrode (Figure 4). Moreover, it validates with a direct measure of neural activity the predominant face-selective activation in the right latFG found in human neuroimaging, the so-called "Fusiform Face Area" (FFA), where the highest face-selective response amplitude was found. Further support that our approach allows measuring local neural sources comes from the observation that the sites where impairments of face perception and/or recognition are observed following intracerebral electrical stimulation, are systematically the sites that record the largest FPVS face-selective response amplitudes among all the implanted electrodes (Jonas et al. 2014; Jonas et al. 2018).

\section{The neural basis of face categorization}

Considering here only the contacts that showed a face-selective response without any house-selective response ( $46.6 \%$ of significant contacts), they were widely distributed across the VOTC, with the largest amplitude responses in the middle section of the right lateral FG as previously observed, i.e. corresponding to the FFA in neuroimaging (Kanwisher et al. 1997), but also in the IOG and the ATL of the right hemisphere (Figure 4). The wide distribution of face-selective responses refers to the numerous brain regions showing a high face-selective response amplitude and/or a high proportion of face-selective responses. These regions include the IOG, the latFG, the antFG, the antOTS, and the antCoS bilaterally, that is, a large a part of the human VOTC running all along the fusiform gyrus from the occipital lobe to the ATL. These regions are thought to form a highly interconnected faceselective network (Gschwind et al. 2011; Pyles et al. 2013; Duchaine and Yovel 2015).

Nevertheless, it is important to stress that a wide distribution as found here or in previous large-scale studies (e.g., Allison et al. 1999 with ECoG) contrasts with the spatially separated clusters identified by fMRI studies (Rossion et al. 2018). Two hypotheses can be 
considered. On the one hand, the present approach may artificially increase the width of the distribution of face-selective responses. Our data visualization, showing maps with the superposition of all significant recording contacts across participants (Figure 3) may enhance the impression of distributed responses (relative to fMRI studies showing maps averaged across participants). This impression is further increased by the large interindividual variability of the location of face-selective areas across individuals (e.g., Rossion et al. 2012; Gao et al. 2018; Zhen et al., 2015). To control for this bias, in the present study, we reported proportion maps of significant contacts (out of total recorded contacts), which were not reported previously (Jonas et al. 2016), highlighting the regions with the highest probability of faceselective responses. Moreover, as discussed above, there is a possibility that some overlap contacts in the present study may capture responses arising from distant source due to volume conduction of the electrical field. The combination of these two factors (data visualization and volume conduction) may explain discrepancies with fMRI findings and offer a potential explanation for rare (among non-significant) and weak face-selective responses in some regions (e.g. MTG/ITG, antMTG/ITG, medFG, i.e., regions lateral or medial to the fusiform gyrus) which are usually not disclosed in fMRI studies. On the other hand, fMRI may not be sensitive enough to record all face-selective responses of the VOTC. FMRI provides only a hemodynamic (indirect) measure of neural activity and therefore smaller, scattered, face-selective responses in the VOTC may be entirely missed by fMRI studies that identify only sufficiently large clusters of activation near blood vessels. In contrast, our approach provides electrophysiological face-selective responses with a very high SNR (FPVS) on small electrodes implanted directly inside the gray matter (SEEG). Moreover, fMRI is affected by severe magnetic susceptibility artefacts that causes a strong signal dropout in the ATL, so that our approach identified many more face-selective regions in the ATL (antCOS, antOTS, antFG, antMTG/ITG). 
In the present study, we report intracranial maps both for amplitude and proportion of significant contacts in the VOTC for the first time (Figure 4). We believe that both types of analyses are important and informative. First, both analyses showed largely overlapping results, thus reinforcing each other. Amplitude and proportion of face-selective responses were maximal in the IOG and all along the fusiform gyrus (latFG, the antFG, the antOTS, and the antCOS bilaterally), reinforcing the extent of the face-selective network, centered on the lateral fusiform gyrus. Second, both types of analyses provide complementary information. For example, while the right latFG showed the largest face-selective response amplitude, the bilateral IOG showed the highest proportion (Figure 4). The right IOG is a region where high face-selective responses are usually observed in fMRI (Haxby et al. 2000; Rossion et al. 2003; Duchaine and Yovel 2015; Grill-Spector et al. 2017), whose lesion can produce prosopagnosia (Rossion et al. 2003; Bouvier and Engel 2006 and whose transient inactivation either by transcranial electrical stimulation (TMS; Pitcher et al. 2007; Ambrus et al., 2017) or intracerebral electrical stimulation (Jonas et al. 2012) can cause difficulties of face identity recognition. The amplitude recorded on a given SEEG contact depends on several factors including the distance between the source and the electrode, the spatial configuration of the cortical source, etc. (Herreras 2016). Therefore, if all conditions are not met to record the largest amplitude possible, the proportion analysis can still identify an important region of the cortical face network independently of the response amplitude.

\section{Face-exclusive responses in the ATL}

Importantly, the wide distribution of face-selective responses refers also to the fact that these responses largely extend to the ATL (Jonas et al. 2016), a region that is difficult to image with fMRI due to strong magnetic artefacts caused by the proximity of the ear canals (Wandell 2011; Axelrod and Yovel 2013; Rossion et al. 2018). In this region, face contacts largely outnumbered house contacts (286 vs. 91). This region also showed the highest 
proportion of so-called "exclusive" contacts (Jonas et al. 2016), i.e. contacts exhibiting significant categorization response $(1.2 \mathrm{~Hz}$ and harmonics) without a general visual stimulation response to other objects at the base frequency (6 Hz and harmonics).

Given the anterior location of these "exclusive" contacts, it was unknown whether they reflected a post-visual-categorization process evoked by any category, or if they were truly part of a face-selective process. For example, it is well known that a post-visual categorization system(s) is capable of monitoring events that differ in frequency, as for example reflected in the robust P300 scalp EEG component located on posterior parietal channels (e.g., Donchin, 1981; Kutas et al. 1977). Consistent with Jonas et al. (2016), here we identified a large proportion of exclusive responses (19.5\% of all significant contacts) that were mostly located in the ATL $(71.11 \%$ of all exclusive contacts). It is important to note that the criteria to define a contact as exclusive was stricter in the present study than in Jonas et al. (2016) (no base response for the preferred category but also no selective and base responses for the nonpreferred category). Contrary to a general oddball detector account, we found that most of the exclusive contacts were responsive to either only faces or only houses $(89.3 \%$ of all exclusive contacts). Notably, most of the exclusive contacts $(71.1 \%)$ responded to faces only.

The current study thus provides additional evidence that response profiles in the ATL are qualitatively different than in the more posterior brain regions. These results suggest that the ATL is involved in the highest stages of face categorization for which faces should be processed independently of the context and for which face information should be shared with other modality-specific regions in order to build a common person-related representation (face-name-voice association, linking information specific to an individual face, etc.). This hypothesis is consistent with a "hub" theory of semantic cognition in which the ATL integrates modality-specific representations into a shared crossmodal representation (Lambon Ralph 2014; Rice et al. 2018). The response profile evolving from category-selective to category- 
exclusive along the posterior-anterior axis may reflect that regions of the temporal lobe become gradually crossmodal as direct connections from early visual areas become sparser and connections with other modality-specific regions increase (voice, name, etc.).

In summary, our large-scale intracerebral recording study provides a global mapping of the human VOTC for selective responses to frequency-tagged faces presented in rapid periodic trains of nonface objects. Face- and house-selective contacts were spatially dissociated along the medial-to-lateral VOTC axis, even across overlap contacts which appeared to measure spatially distinct populations of neurons responding selectively to each category, and this dissociation was present up to the ATL. A high proportion of intracerebral recording contacts in the ATL show exclusive responses to faces, indicating that this region is particularly important for higher-order face-related processes independent of general visual responses. Altogether, these observations shed light on the neural basis of human face categorization and strengthen the validity of the frequency-tagging approach coupled with intracerebral recordings in epileptic patients to understand visual categorization and human brain function in general.

\section{Funding}

The research has been funded by a LUE (Lorraine Universite d'Excellence) program, a 2018 project from the Région Grand Est, and a FNRS EOS project (ID: 30991544). 


\section{References}

Allison T, McCarthy G, Nobre, A., Puce A, Belger A. 1994. Human extrastriate visual cortex and the perception of faces, words, numbers, and colors. Cerebral cortex, 4: 544-554.

Allison T, Puce A, Spencer DD, McCarthy G. 1999. Electrophysiological studies of human face perception. I: Potentials generated in occipitotemporal cortex by face and non-face stimuli. Cerebral cortex, 9: 415-430.

Ambrus, GG, Dotzer, M, Schweinberger, SR, Kovács, G. 2017. The occipital face area is causally involved in the formation of identity-specific face representations. Brain Structure and Function. 222: 4271-4282.

Axelrod V, Yovel G. 2013. The challenge of localizing the anterior temporal face area: a possible solution. Neuroimage, 81: 371-380.

Bastin J, Vidal JR, Bouvier S, Perrone-Bertolotti M, Bénis D, Kahane P, David O, Lachaux JP, Epstein RA. 2013. Temporal components in the parahippocampal place area revealed by human intracerebral recordings. Journal of Neuroscience, 33:10123-10131.

Bédos-Ulvin LB, Jonas J, Brissart H, Colnat-Coulbois S, Thiriaux A, Vignal JP, Maillard L. 2017. Intracerebral stimulation of left and right ventral temporal cortex during object naming. Brain and language, 175: 71-76.

Bouvier SE, Engel SA. 2006. Behavioral deficits and cortical damage loci in cerebral achromatopsia. Cerebral cortex, 16:183-191.

Buzsáki G, Anastassiou CA, Koch C. 2012. The origin of extracellular fields and currentsEEG, ECoG, LFP and spikes. Nature reviews neuroscience, 13: 407. 
Carpenter B, Gelman A, Hoffman MD, Lee D, Goodrich B, Betancourt M, Brubaker M, Guo J, Li P, Riddell A. 2017. Stan: A probabilistic programming language. Journal of Statistical Software, 76.

Collins JA, Koski JE, Olson IR. 2016. More than meets the eye: The merging of perceptual and conceptual knowledge in the anterior temporal face area. Frontiers in human neuroscience, 10: 189.

Collins JA, Olson IR. 2014. Beyond the FFA: the role of the ventral anterior temporal lobes in face processing. Neuropsychologia, 61: 65-79.

Cox RW. 1996. AFNI: software for analysis and visualization of functional magnetic resonance neuroimages. Computers and Biomedical research, 29: 162-173.

Ding SL, Van Hoesen GW, Cassell MD, Poremba A. 2009. Parcellation of human temporal polar cortex: a combined analysis of multiple cytoarchitectonic, chemoarchitectonic, and pathological markers. Journal of Comparative Neurology, 514: 595-623.

Donchin E. 1981. Surprise!... surprise?. Psychophysiology, 18: 493-513.

Downing PE, Chan AY, Peelen MV, Dodds CM, Kanwisher N. 2005. Domain specificity in visual cortex. Cerebral cortex, 16: 1453-1461.

Dubey A, Ray S. 2019. Cortical Electrocorticogram (ECoG) is a local signal. Journal of Neuroscience, 39: 4299-4311.

Duchaine B, Yovel G. 2015. A revised neural framework for face processing. Annual Review of Vision Science, 1: 393-416. 
Engell AD, McCarthy G. 2010. The relationship of gamma oscillations and face-specific ERPs recorded subdurally from occipitotemporal cortex. Cerebral cortex, 21: 1213-1221.

Epstein RA, Bar M, Kveraga K. 2014. Neural systems for visual scene recognition. Scene vision: 105-134.

Epstein R, Kanwisher N. 1998. A cortical representation of the local visual environment. Nature, 392: 598.

Gainotti G. 2013. Is the right anterior temporal variant of prosopagnosia a form of 'associative prosopagnosia' or a form of 'multimodal person recognition disorder'? Neuropsychology Review, 23: 99-110.

Gao X, Gentile F, Rossion B. 2018. Fast periodic stimulation (FPS): a highly effective approach in fMRI brain mapping. Brain Structure and Function, 223: 2433-2454.

Ghuman AS, Brunet NM, Li Y, Konecky RO, Pyles JA, Walls SA, Destefino V, Wang W, Richardson RM. 2014. Dynamic encoding of face information in the human fusiform gyrus. Nature communications, 5: 5672.

Gomez J, Pestilli F, Witthoft N, Golarai G, Liberman A, Poltoratski S, Yoon J, Grill-Spector K. 2015. Functionally defined white matter reveals segregated pathways in human ventral temporal cortex associated with category-specific processing. Neuron, 85: 216-227.

Grill-Spector K, Weiner KS, Kay K, Gomez J. 2017. The functional neuroanatomy of human face perception. Annual review of vision science, 3: 167-196. 
Gschwind M, Pourtois G, Schwartz S, Van De Ville D, Vuilleumier P. 2011. White-matter connectivity between face-responsive regions in the human brain. Cerebral cortex, 22: 15641576.

Halgren E, Baudena P, Heit G, Clarke M, Marinkovic K. 1994. Spatio-temporal stages in face and word processing. 1. Depth recorded potentials in the human occipital and parietal lobes. Journal of Physiology-Paris, 88: 1-50.

Haxby JV, Hoffman EA, Gobbini MI. 2000. The distributed human neural system for face perception. Trends in cognitive sciences, 4: 223-233.

Herreras O. 2016. Local field potentials: myths and misunderstandings. Frontiers in neural circuits, 10: 101.

Jacques C, Retter TL, Rossion B. 2016. A single glance at natural face images generate larger and qualitatively different category-selective spatio-temporal signatures than other ecologically-relevant categories in the human brain. Neurolmage, 137: 21-33.

Jacques C, Witthoft N, Weiner KS, Foster BL, Rangarajan V, Hermes D, Miller KJ, Parvizi J, Grill-Spector K. 2016. Corresponding ECoG and fMRI category-selective signals in human ventral temporal cortex. Neuropsychologia, 83: 14-28.

Jonas J, Brissart H, Hossu G, Colnat-Coulbois S, Vignal JP, Rossion B, Maillard L. 2018. A face identity hallucination (palinopsia) generated by intracerebral stimulation of the faceselective right lateral fusiform cortex. Cortex, 99: 296-310. 
Jonas J, Descoins M, Koessler L, Colnat-Coulbois S, Sauvée M, Guye M, ... Maillard L. 2012. Focal electrical intracerebral stimulation of a face-sensitive area causes transient prosopagnosia. Neuroscience, 222: 281-288.

Jonas J, Jacques C, Liu-Shuang J, Brissart H, Colnat-Coulbois S, Maillard L. Rossion B. 2016. A face-selective ventral occipito-temporal map of the human brain with intracerebral potentials. Proceedings of the National Academy of Sciences, 113: E4088-E4097.

Jonas J, Rossion B, Krieg J, Koessler L, Colnat-Coulbois S, Vespignani H, Jacques C, Vignal JP, Brissart H, Maillard L. 2014. Intracerebral electrical stimulation of a face-selective area in the right inferior occipital cortex impairs individual face discrimination. Neuroimage, 99: 487497.

Kadipasaoglu CM, Conner CR, Whaley ML, Baboyan VG, Tandon N. 2016. Categoryselectivity in human visual cortex follows cortical topology: a grouped icEEG study. PloS one, 11: e0157109.

Kanwisher N, McDermott J, Chun, MM. 1997. The fusiform face area: a module in human extrastriate cortex specialized for face perception. Journal of neuroscience, 17: 4302-4311.

Katzner S, Nauhaus I, Benucci A, Bonin V, Ringach DL, Carandini M. 2009. Local origin of field potentials in visual cortex. Neuron, 61: 35-41.

Koessler L, Maillard L, Benhadid A, Vignal JP, Felblinger J, Vespignani H, Braun M. 2009. Automated cortical projection of EEG sensors: anatomical correlation via the international 1010 system. Neuroimage, 46: 64-72. 
Kruschke JK. 2013. Bayesian estimation supersedes the $t$ test. Journal of Experimental Psychology: General, 142: 573.

Kruschke J. 2014. Doing Bayesian data analysis: A tutorial with R, JAGS, and Stan. Academic Press.

Kutas M, McCarthy G. Donchin E. 1977. Augmenting mental chronometry: the P300 as a measure of stimulus evaluation time. Science, 197: 792-795.

Lambon Ralph MA. 2014. Neurocognitive insights on conceptual knowledge and its breakdown. Philosophical Transactions of the Royal Society B: Biological Sciences, 369: 20120392.

Lochy A, Jacques C, Maillard L, Colnat-Coulbois S, Rossion B, Jonas J. 2018. Selective visual representation of letters and words in the left ventral occipito-temporal cortex with intracerebral recordings. Proceedings of the National Academy of Sciences, 115: E7595E7604.

Meadows JC. 1974. The anatomical basis of prosopagnosia. Journal of Neurology, Neurosurgery, and Psychiatry. 37: 489-501.

Nasr S, Liu N, Devaney KJ, Yue X, Rajimehr R, Ungerleider LG, Tootell RB. 2011. Sceneselective cortical regions in human and nonhuman primates. Journal of Neuroscience, 31: $13771-13785$.

Pitcher D, Walsh V, Yovel G, Duchaine B. 2007. TMS evidence for the involvement of the right occipital face area in early face processing. Current Biology, 17: 1568-1573. 
Puce A, Allison T, Gore JC, McCarthy G. 1995. Face-sensitive regions in human extrastriate cortex studied by functional MRI. Journal of neurophysiology, 74: 1192-1199.

Pyles JA, Verstynen TD, Schneider W, Tarr MJ. 2013. Explicating the face perception network with white matter connectivity. PloS one, 8: e61611.

Quek GL, Rossion B. 2017. Category-selective human brain processes elicited in fast periodic visual stimulation streams are immune to temporal predictability. Neuropsychologia, 104: 182200.

Regan D. 1989. Human brain electrophysiology: Evoked potentials and evoked magnetic fields in science and medicine. Amsterdam, the Netherlands: Elsevier.

Rice GE, Caswell H, Moore P, Hoffman P, Lambon Ralph MA. 2018. The roles of left versus right anterior temporal lobes in semantic memory: a neuropsychological comparison of postsurgical temporal lobe epilepsy patients. Cerebral Cortex, 28: 1487-1501.

Rossion B. 2014. Understanding face perception by means of prosopagnosia and neuroimaging. Front Biosci (Elite Ed), 6: 258-307.

Rossion B, Caldara R, Seghier M, Schuller AM, Lazeyras F, Mayer E. 2003. A network of occipito-temporal face-sensitive areas besides the right middle fusiform gyrus is necessary for normal face processing. Brain, 126: 2381-2395.

Rossion B, Hanseeuw B, Dricot L. 2012. Defining face perception areas in the human brain: a large-scale factorial fMRI face localizer analysis. Brain and cognition, 79: 138-157. 
Rossion B, Jacques C, Jonas J. 2018. Mapping face categorization in the human ventral occipitotemporal cortex with direct neural intracranial recordings. Annals of the New York Academy of Sciences, 1426: 5-24.

Salado AL, Koessler L, De Mijolla G, Schmitt E, Vignal JP, Civit T, Tyvaert L, Jonas J, Maillard LG, Colnat-Coulbois S. 2017. sEEG is a safe procedure for a comprehensive anatomic exploration of the insula: A retrospective study of 108 procedures representing 254 transopercular insular electrodes. Operative Neurosurgery, 14: 1-8.

Spiridon M, Fischl B, Kanwisher N. 2006. Location and spatial profile of category-specific regions in human extrastriate cortex. Human brain mapping, 27: 77-89.

Tanji K, Iwasaki M, Nakasato N, Suzuki K. 2012. Face specific broadband electrocorticographic spectral power change in the rhinal cortex. Neuroscience letters, 515 : 66-70.

Tsao DY, Moeller S, Freiwald WA. 2008. Comparing face patch systems in macaques and humans. Proceedings of the National Academy of Sciences, $105: 19514-19519$.

Tootell RB, Devaney KJ, Young JC, Postelnicu G, Rajimehr R, Ungerleider LG. 2008. fMRI mapping of a morphed continuum of 3D shapes within inferior temporal cortex. Proceedings of the National Academy of Sciences, 105: 3605-3609.

Vidal JR, Ossandón T, Jerbi K, Dalal SS, Minotti L, Ryvlin P, Kahane P, Lachaux, JP. 2010. Category-specific visual responses: an intracranial study comparing gamma, beta, alpha, and ERP response selectivity. Frontiers in human neuroscience, 4, 195. 
Yoshor D, Bosking WH, Ghose GM, Maunsell JH. 2006. Receptive fields in human visual cortex mapped with surface electrodes. Cerebral cortex, 17: 2293-2302.

Yoshor D, Ghose GM, Bosking WH, Sun P, Maunsell JH. 2007. Spatial attention does not strongly modulate neuronal responses in early human visual cortex. Journal of Neuroscience, 27: 13205-13209.

Van Hoesen GW. 1995. Anatomy of the medial temporal lobe. Magnetic resonance imaging, 13: 1047-1055.

van Hoesen GW, Augustinack JC, Dierking J, Redman SJ, Thangavel, R. 2000. The parahippocampal gyrus in Alzheimer's disease: clinical and preclinical neuroanatomical correlates. Annals of the New York Academy of Sciences, 911: 254-274.

Wandell BA. 2011. The neurobiological basis of seeing words. Annals of the New York Academy of Sciences, 1224: 63-80.

Weiner KS, Barnett, MA, Witthoft N, Golarai G, Stigliani A, Kay KN, ... Grill-Spector K. 2018. Defining the most probable location of the parahippocampal place area using cortex-based alignment and cross-validation. Neuroimage, 170: 373-384.

Weiner, KS., Grill-Spector, K. 2010. Sparsely-distributed organization of face and limb activations in human ventral temporal cortex. Neuroimage, 52: 1559-1573.

Wennberg R. 2010. On electrical potentials observed at a distance from intracranial electrode contacts. Clinical neurophysiology: official journal of the International Federation of Clinical Neurophysiology, 121: 259. 
Zaveri HP, Duckrow RB, Spencer SS. 2009. Concerning the observation of an electrical potential at a distance from an intracranial electrode contact. Clinical neurophysiology, 10: 1873-1875.

Zhen Z, Yang Z, Huang L, Kong XZ, Wang X, Dang X, ... Liu J. 2015. Quantifying interindividual variability and asymmetry of face-selective regions: a probabilistic functional atlas. Neuroimage, 113: 13-25.

\section{Supplementary Figures}

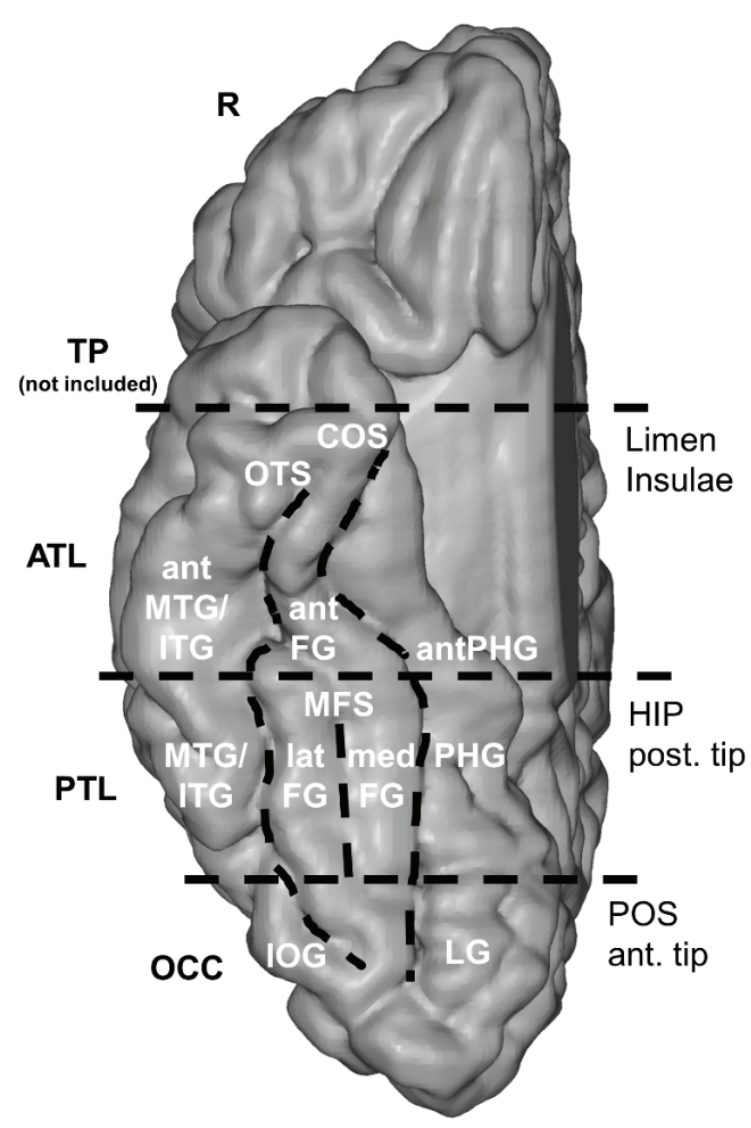


Figure S1. Schematic representation of the parcellation scheme used to determine the anatomical label of each contact. Anatomical regions were defined in each individual hemisphere according to major anatomical landmarks. The ventral temporal sulci (COS, OTS, and midfusiform sulcus, i.e., MFS) serve as medial/lateral borders of regions, whereas two coronal reference planes containing anatomical landmarks (posterior tip of the hippocampus, i.e., HIP and anterior tip of the parieto-occipital sulcus, i.e., POS) serve as an anterior/posterior boundary for each region. We considered contacts in the ATL if they were located anteriorly to the posterior tip of the hippocampus. Note that we did not include in our analyses contacts in the temporal pole (TP), i.e., anterior to the limen insulae. The schematic locations of these anatomical structures are shown on a reconstructed cortical surface of the Colin27 brain.
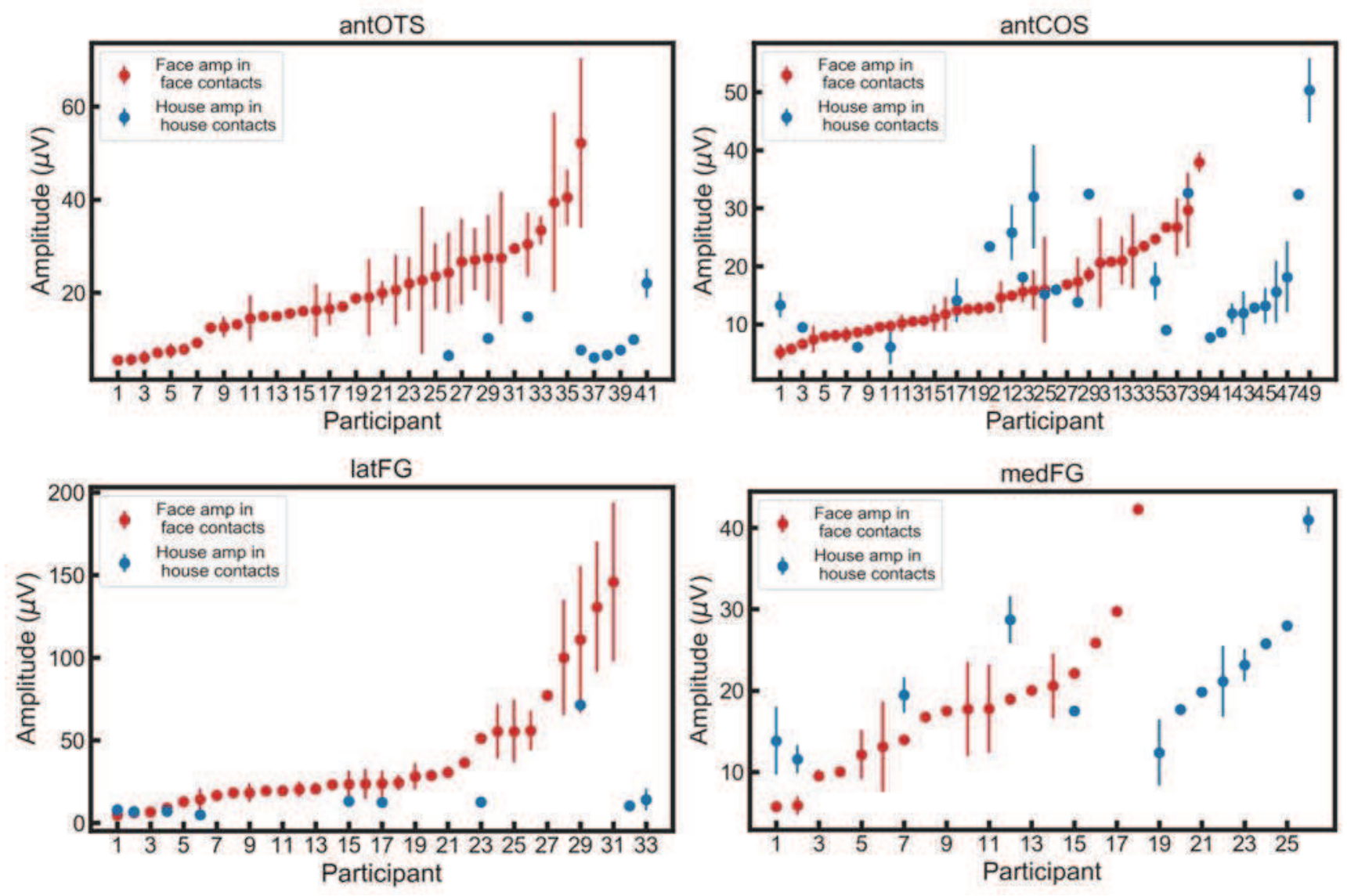

Figure S2. Individual participant analysis in four anatomical regions (antOTS, antCOS, latFG, medFG). For each participant, we averaged the selective amplitude across face or house contacts belonging to the same anatomical region. Each dot therefore represents the mean selective amplitude for face or house contacts in a single region of a single participant. Error bars represents SEMs. The participants are ranked from lowest to highest average amplitudes for faces (i.e., participant number does not match across the different panels). As expected, there is variability in terms of amplitude across participants, which could be due several factors: contacts distance to the source, dipole orientation, size of the generator (i.e., of the functional area), etc. 